\title{
Combined search for the quarks of a sequential fourth generation
}

\author{
S. Chatrchyan et al. ${ }^{*}$ \\ (CMS Collaboration)
}

(Received 5 September 2012; published 12 December 2012)

\begin{abstract}
Results are presented from a search for a fourth generation of quarks produced singly or in pairs in a data set corresponding to an integrated luminosity of $5 \mathrm{fb}^{-1}$ recorded by the CMS experiment at the LHC in 2011. A novel strategy has been developed for a combined search for quarks of the up and down type in decay channels with at least one isolated muon or electron. Limits on the mass of the fourth-generation quarks and the relevant Cabibbo-Kobayashi-Maskawa matrix elements are derived in the context of a simple extension of the standard model with a sequential fourth generation of fermions. The existence of mass-degenerate fourth-generation quarks with masses below $685 \mathrm{GeV}$ is excluded at $95 \%$ confidence level for minimal off-diagonal mixing between the third- and the fourth-generation quarks. With a mass difference of $25 \mathrm{GeV}$ between the quark masses, the obtained limit on the masses of the fourth-generation quarks shifts by about $\pm 20 \mathrm{GeV}$. These results significantly reduce the allowed parameter space for a fourth generation of fermions.
\end{abstract}

DOI: 10.1103/PhysRevD.86.112003

PACS numbers: 13.85.Rm, 12.60.-i, 14.65.Jk

\section{INTRODUCTION}

The existence of three generations of fermions has been firmly established experimentally [1]. The possibility of a fourth generation of fermions has not been excluded, although it is strongly constrained by precision measurements of electroweak observables. These observables are mainly influenced by the mass differences between the fourth-generation leptons or quarks. In particular, scenarios with a mass difference between the fourth-generation quarks smaller than the mass of the $W$ boson are preferred, and even fourth-generation quarks with degenerate masses are allowed $[2,3]$.

A new generation of fermions requires not only the existence of two additional quarks and two additional leptons, but also an extension of the Cabibbo-KobayashiMaskawa (CKM) [4,5] and Pontecorvo-Maki-NakagawaSakata [6,7] matrices. New CKM (quark mixing) and Pontecorvo-Maki-Nakagawa-Sakata (lepton mixing) matrix elements are constrained by the requirement of consistency with electroweak precision measurements [8].

Previous searches at hadron colliders have considered either pair production or single production of one of the fourth-generation quarks [9-15]. The most stringent limits exclude the existence of a down-type (up-type) fourthgeneration quark with a mass below 611 (570) $\mathrm{GeV}$ $[14,15]$. These limits on the quark mass values enter a region where the coupling of fourth-generation quarks to the Higgs field becomes large and perturbative calculations for the weak interaction start to fail, assuming the absence

*Full author list given at the end of the article.

Published by the American Physical Society under the terms of the Creative Commons Attribution 3.0 License. Further distribution of this work must maintain attribution to the author(s) and the published article's title, journal citation, and DOI. of other phenomena beyond the standard model [16]. To increase the sensitivity and to use a consistent approach while searching for a new generation of quarks, we have developed a simultaneous search for the up-type and downtype fourth-generation quarks, based on both the electroweak and strong production mechanisms.

If a fourth generation of quarks exists, their production cross sections and decay branching fractions will be governed by an extended $4 \times 4$ CKM matrix, $V_{\text {CKM }}^{4 \times 4}$, in which we denote the up- and down-type fourth-generation quarks as $t^{\prime}$, and $b^{\prime}$, respectively. For simplicity, we assume a model with one free parameter, $A$, where $0 \leq A \leq 1$ :

$$
\begin{aligned}
V_{\mathrm{CKM}}^{4 \times 4} & =\left(\begin{array}{cccc}
V_{u d} & V_{u s} & V_{u b} & V_{u b^{\prime}} \\
V_{c d} & V_{c s} & V_{c b} & V_{c b^{\prime}} \\
V_{t d} & V_{t s} & V_{t b} & V_{t b^{\prime}} \\
V_{t^{\prime} d} & V_{t^{\prime} s} & V_{t^{\prime} b} & V_{t^{\prime} b^{\prime}}
\end{array}\right) \\
& =\left(\begin{array}{cccc}
\mathcal{O}(1) & \mathcal{O}(0) & \mathcal{O}(0) & 0 \\
\mathcal{O}(0) & \mathcal{O}(1) & \mathcal{O}(0) & 0 \\
\mathcal{O}(0) & \mathcal{O}(0) & \sqrt{A} & \sqrt{1-A} \\
0 & 0 & -\sqrt{1-A} & \sqrt{A}
\end{array}\right) .
\end{aligned}
$$

The complex phases are not shown for clarity. Within this model, mixing is allowed only between the third and the fourth generations. This is a reasonable assumption since the mixing between the third and the first two generations is observed to be small [17]. However, the limits presented in this paper would be too stringent if there is a fourth generation that mixes only with the first two generations, or the size of the mixing with the third generation is about the same as the mixing with the first two generations.

With this search, we set limits on the masses of the fourthgeneration quarks as a function of $A$. Since $\sqrt{A}=\left|V_{t b}\right|$, the lower limit of $\left|V_{t b}\right|>0.81$ from the single-top production 
cross section measurements [18] translates into a lower limit on the mixing between the third- and fourthgeneration quarks in our model of $A>0.66$.

Using the data collected from $\sqrt{s}=7 \mathrm{TeV}$ protonproton collisions at the Large Hadron Collider (LHC), we search for fourth-generation quarks that are produced in pairs, namely $b^{\prime} \bar{b}^{\prime}$ and $t^{\prime} \bar{t}^{\prime}$, or through electroweak production, in particular $t b^{\prime}, t^{\prime} b$, and $t^{\prime} b^{\prime}$, where the charges are omitted in the notation. While the cross sections of the pair production processes do not depend on the value of $A$, the production cross sections of the $t b^{\prime}$ and $t^{\prime} b$ processes depend linearly on $(1-A)$, and the single-top and $t^{\prime} b^{\prime}$ cross sections on $A$.

We assume the $t^{\prime}$ and $b^{\prime}$ masses to be degenerate within $25 \mathrm{GeV}$. In the case they are degenerate, they will decay in $100 \%$ of the cases to the third-generation quarks, since the decay of one fourth-generation quark to the other is kinematically not allowed. However, even for nonzero mass differences, the branching fractions of the $t^{\prime} \rightarrow b W$ and the $b^{\prime} \rightarrow t W \rightarrow(b W) W$ decays are close to $100 \%$, provided that the mass difference is small [19]. For instance, for a mass splitting of $25 \mathrm{GeV}$, and for $V_{t^{\prime} b^{\prime}}=0.005$ (which would correspond to $A=0.99975$ in our model), less than $5 \%$ of the decays will be $b^{\prime} \rightarrow t^{\prime} W^{*}$ (in the case $m_{t^{\prime}}<m_{b^{\prime}}$ ) or $t^{\prime} \rightarrow b^{\prime} W^{*}$ (in the case $m_{t^{\prime}}>m_{b^{\prime}}$ ). For larger values of $V_{t^{\prime} b}$, the branching fractions of $b^{\prime} \rightarrow t^{\prime} W^{*}$ (or $t^{\prime} \rightarrow b^{\prime} W^{*}$ ) decrease even further. Therefore, the decay chains remain unchanged as long as the mass splitting is relatively small. We expect the following final states:

(i) $t^{\prime} b \rightarrow b W b$

(ii) $t^{\prime} t^{\prime} \rightarrow b W b W$

(iii) $b^{\prime} t \rightarrow t W b W \rightarrow b W W b W$;

(iv) $b^{\prime} t^{\prime} \rightarrow t W b W \rightarrow b W W b W$;

(v) $b^{\prime} \bar{b}^{\prime} \rightarrow t W t W \rightarrow b W W b W W$.

These decay chains imply that two jets from $b$ quarks and one to four $W$ bosons are expected in the final state for fourth-generation quarks produced both singly and in pairs. The $W$ bosons decay to either hadronic or leptonic final states. Events with either one isolated lepton (muon or electron) or two same-sign dileptons or three leptons are selected. The different production processes are classified according to the number of observed $W$ bosons.

\section{THE COMPACT MUON SOLENOID DETECTOR}

The central feature of the Compact Muon Solenoid (CMS) detector is a superconducting solenoid, $13 \mathrm{~m}$ in length and $6 \mathrm{~m}$ in internal diameter, providing an axial magnetic field of $3.8 \mathrm{~T}$. The inside of the solenoid is equipped with various particle detection systems. Charged particle trajectories are measured by a silicon pixel and strip tracker, covering $0<\phi<2 \pi$ in azimuth and $|\eta|<2.5$, where the pseudorapidity $\eta$ is defined as $-\ln [\tan (\theta / 2)]$, and $\theta$ is the polar angle of the trajectory with respect to the anticlockwise-beam direction. A crystal electromagnetic calorimeter and a brass/scintillator hadron calorimeter surround the tracking volume and provide high-resolution energy and direction measurements of electrons, photons, and hadronic jets. Muons are measured in gas-ionization detectors embedded in the steel return yoke outside the solenoid. The CMS detector also has extensive forward calorimetry covering up to $|\eta|<5$. The detector is nearly hermetic, allowing for energy balance measurements in the plane transverse to the beam directions. A two-tier trigger system selects the most interesting proton collision events for use in physics analysis. A more detailed description of the CMS detector can be found elsewhere [20].

\section{EVENT SELECTION AND SIMULATION}

The search for the fourth-generation quarks is performed using the $\sqrt{s}=7 \mathrm{TeV}$ proton-proton collisions recorded by the CMS experiment at the LHC. We have analyzed the full data set collected in 2011 corresponding to an integrated luminosity of $(5.0 \pm 0.1) \mathrm{fb}^{-1}$. Events are selected with a trigger requiring an isolated muon or electron, where the latter is accompanied by at least one jet identified as a $b$ jet. The muon system, the calorimetry, and the tracker are used for the particle-flow event reconstruction [21]. Jets are reconstructed using the anti- $k_{\mathrm{T}}$ algorithm [22] with a size parameter of 0.5. Events are further selected with at least one high-quality isolated muon or electron with a transverse momentum $\left(p_{\mathrm{T}}\right)$ exceeding $40 \mathrm{GeV}$ in the acceptance range $|\eta|<2.1$ for muons and $|\eta|<2.5$ for electrons. The relative isolation, $I_{\text {rel }}$, is calculated from the other particle-flow particles within a cone of $\Delta R=$ $\sqrt{(\Delta \phi)^{2}+(\Delta \eta)^{2}}<0.4$ around the axis of the lepton. It is defined as $I_{\text {rel }}=\left(E_{\mathrm{T}}^{\text {charged }}+E_{\mathrm{T}}^{\text {photon }}+E_{\mathrm{T}}^{\text {neutral }}\right) / p_{\mathrm{T}}$, where $E_{\mathrm{T}}^{\text {charged }}$ and $E_{\mathrm{T}}^{\text {photon }}$ are the transverse energies deposited by charged hadrons and photons, respectively, and $E_{\mathrm{T}}^{\text {neutral }}$ is the transverse energy deposited by neutral particles other than photons. We identify muons and electrons as isolated when $I_{\text {rel }}<0.125$ and $I_{\text {rel }}<0.1$, respectively. The requirement on the relative isolation for electrons is tighter than for muons because the backgrounds for electrons are higher than for muons. Electron candidates in the transition region between electromagnetic calorimeter barrel and end cap $(1.44<|\eta|<1.57)$ are excluded because the reconstruction of an electron object in this region is not optimal. We require a missing transverse momentum $\mathscr{E}_{\mathrm{T}}$ of at least $40 \mathrm{GeV}$. The $\mathbb{E}_{\mathrm{T}}$ is calculated as the absolute value of the vector sum of the $p_{\mathrm{T}}$ of all reconstructed objects. Jets are required to have a $p_{\mathrm{T}}>30 \mathrm{GeV}$. The jet energies are corrected to establish a uniform response of the calorimeter in $\eta$ and a calibrated absolute response in $p_{\mathrm{T}}$. Furthermore, a correction is applied to take into account the energy clustered in jets due to additional proton interactions in the same bunch crossing. 
The observed data are compared to simulated data generated with POWHEG $301[23,24]$ for the single-top process, PYTHIA 6.4.22 [25] for the diboson processes, and MADGRAPH 5.1.1 [26] for the signal and other standard model processes. The POWHEG and MADGRAPH generators are interfaced with PYTHIA for the decay of the particles as well as the hadronization and the implementation of a CMS custom underlying event tuning (tune Z2) [27]. The matching of the matrix-element partons to the parton showers is obtained using the MLM matching algorithm [28]. The CTEQ6L1 leading-order (LO) parton distributions are used in the event generation [29]. The generated events are passed through the CMS detector simulation based on GEANT4 [30], and then processed by the same reconstruction software as the collision data. The simulated events are reweighted to match the observed distribution of the number of simultaneous proton interactions. For the full data set collected in 2011, we observe on average about nine interactions in each event. We smear the jet energies in the simulation to match the resolutions measured with data [31]. At least one of the jets within the tracker acceptance $(|\eta|<2.4)$ needs to be identified as a $b$ jet. For the $b$-jet identification, we require the signed impact parameter significance of the third track in the jet (sorted by decreasing significance) to be larger than a value chosen such that the probability for a light quark jet to be misidentified as a $b$ jet is about $1 \%$. We apply scale factors measured from data to the simulated events to take into account the different $b$-jet efficiency and the different probability that a light quark or gluon is identified as a $b$ jet in data and simulation [32].

The top-quark pair as well as the $W$ and $Z$ production cross section values used in the analysis correspond to the measured values from CMS $[33,34]$. We use the predicted cross section values for the single-top, $t \bar{t}+W, t \bar{t}+Z$, and same-sign $W W$ processes [35-38]. The cross section values for the diboson production are obtained with the MCFM next-to-leading-order parton-level integrator $[39,40]$.

For the pair-production of the fourth-generation quarks, we use the approximate next-to-next-to-leading-order cross section values from Ref. [41]. For the electroweak production processes mentioned above, we rescale the next-to-leading-order cross sections at $14 \mathrm{TeV}$ [42] to $7 \mathrm{TeV}$ using a scale factor defined as the ratio of the LO cross section at $7 \mathrm{TeV}$ and the $\mathrm{LO}$ cross section at $14 \mathrm{TeV}$ as obtained by the MADGRAPH event generator. The resulting production cross sections are maximal, hence assuming $\left|V_{t b^{\prime}}\right|=\left|V_{t^{\prime} b}\right|=\left|V_{t^{\prime} b^{\prime}}\right|=1$, and are rescaled according to the value of $A$.

\section{EVENT CLASSIFICATION}

Different channels are defined according to the number of $W$ bosons in the final state. Given that the $t^{\prime}$ decay mode is the same as the top-quark decay mode, the $t^{\prime} b$ and $t^{\prime} t^{\prime}$ processes will yield signatures that are very similar to, respectively, the single-top and $t \bar{t}$ processes in the standard model. We select these processes through the single-lepton decay channel. In the signal final states that contain a $b^{\prime}$ quark, we expect three or four $W$ bosons. If two or more of these $W$ bosons decay to leptons, we may have events with two leptons of the same charge or with three charged leptons. Although the branching fraction of these decays is small compared to that of other decay channels, these final states are very interesting because of the low background that is expected from standard model processes.

\section{A. The single-electron and single-muon decay channels}

On top of the aforementioned event selection criteria, we veto events with additional electrons or muons with $I_{\text {rel }}<$ 0.2 and $p_{\mathrm{T}}>10 \mathrm{GeV}$ for muons and $p_{\mathrm{T}}>15 \mathrm{GeV}$ for electrons. We divide the selected single-lepton events into different subsamples according to the signal final states. Therefore, we define a procedure to count the number of $W$-boson candidates. Each event has at least one $W$ boson that decays to leptons, consistent with the requirements of an isolated lepton and a large missing transverse momentum from the neutrino, which escapes detection. The decays of $W$ bosons to $q \bar{q}$ final states are reconstructed with the following procedure. For each event, we have a collection of selected jets used as input for the reconstruction of the $W$-boson candidates. The one or two jets that are identified as $b$ jets are removed from the collection. $W$-boson candidates are constructed from all possible pairs of the remaining jets in the collection. We use both the expected mass, $m_{W}^{\mathrm{fit}}=84.3 \mathrm{GeV}$, and the width, $\sigma_{m W}^{\mathrm{fit}}=$ $9.6 \mathrm{GeV}$, from a Gaussian fit to the reconstructed mass distribution of jet pairs from the decay of a $W$ boson in simulated $t \bar{t}$ events. The $W$-boson candidate with a mass that matches the value of $m_{W}^{\text {fit }}$ best is chosen as a $W$ boson if its mass is within a $\pm 1 \sigma_{m W}^{\mathrm{fit}}$ window around $m_{W}^{\mathrm{fit}}$. The jet pair that provided the hadronically decaying $W$ boson is removed from the collection, and the procedure is repeated until no more candidates are found for $W$ bosons decaying to jets. Different exclusive subsamples are defined according to the number of $b$ jets (exactly one or at least two) and the number of $W$-boson candidates (one, two, three, and at least four). There are seven subsamples, because we do not consider the subsample with only one $b$ jet and one $W$ boson. The subsample with two $b$ jets and one $W$ boson is dominated by singly produced $t^{\prime}$ events. In this subsample, we apply a veto for additional jets with a transverse momentum exceeding $30 \mathrm{GeV}$. Furthermore, since $b \bar{b}$ background tends to have jets which are produced backto-back with balanced $p_{T}$, we remove this background by requiring $\Delta \phi\left(j_{1}, j_{2}\right)<\frac{\pi}{2}+\pi\left(p_{\mathrm{T}}^{j_{1}}-p_{\mathrm{T}}^{j_{2}}\right) /\left(p_{\mathrm{T}}^{j_{1}}+p_{\mathrm{T}}^{j_{2}}\right)$.

Table I summarizes the requirements that define the different single-lepton decay subsamples, after the criteria on the $\mathscr{E}_{\mathrm{T}}$, and the lepton and jet $p_{\mathrm{T}}$ and $\eta$ are applied.

Table II shows the observed and predicted event yields. After the selection criteria, the dominant background 
TABLE I. Overview of the event selection requirements defining the different subsamples in the single-lepton decay channel. The single-lepton decay channel is divided in seven different subsamples according to the number of $b$ jets and the number of $W$-boson candidates.

\begin{tabular}{cccc}
\hline \hline \multicolumn{4}{c}{ Single-lepton decay channel } \\
$1 W$ & $2 W$ & $3 W$ & $4 W$ \\
\hline$=2$ jets & $\geq 4$ jets & $\geq 6$ jets & $\geq 8$ jets \\
$=2 b$ jets & \multicolumn{4}{c}{ either $=1$ or $\geq 2 b$ jets } \\
$\Delta \phi\left(j_{1}, j_{2}\right)$ requirement & $1 W \rightarrow q \bar{q}$ & $2 W \rightarrow q \bar{q}$ & $3 W \rightarrow q \bar{q}$ \\
\hline \hline
\end{tabular}

contributions result from the production of top-quark pairs, $W+$ jets, and single top. Other processes with very small contributions to the total background are $Z+$ jets and diboson production, and also top-quark pairs produced in association with a $W$ or $Z$ boson. The combined event yield of these processes is about $1 \%$ of the total standard-model contribution. The multijet background is found to be negligible in each of the subsamples. The reason is the requirements of an isolated muon or electron with $p_{\mathrm{T}}>40 \mathrm{GeV}$, a missing transverse momentum of $40 \mathrm{GeV}$, and at least one jet identified as a $b$ jet. Data and simulation are found to agree within the combined statistic and systematic uncertainties.

\section{B. The same-sign dilepton and trilepton decay channels}

The transverse momentum of at least one of the leptons in the multilepton channel is required to be larger than $40 \mathrm{GeV}$, while the threshold is reduced to $20 \mathrm{GeV}$ for additional leptons. Events with two muons or electrons with a mass within $10 \mathrm{GeV}$ of the $Z$-boson mass are rejected to reduce the standard model background with $Z$ bosons in the final state. We require at least four jets for the same-sign dilepton events. In the case of the trilepton events, the minimum number of required jets is reduced to two. Table III summarizes the event selection requirements defining the same-sign dilepton and trilepton decay
TABLE III. Overview of the event selection requirements specific to the same-sign dilepton and trilepton decay channels.

\begin{tabular}{cc}
\hline \hline Same-sign dilepton & Trilepton \\
\hline$=2$ isolated leptons with same sign & $=3$ isolated leptons \\
$\geq 4$ jets $\left(p_{\mathrm{T}}>30 \mathrm{GeV},|\eta|<2.4\right)$ & $\geq 2$ jets $\left(p_{\mathrm{T}}>30 \mathrm{GeV}\right.$, \\
& $|\eta|<2.4)$ \\
$\geq 1 b$ jet & $\geq 1 b$ jet \\
\hline \hline
\end{tabular}

channels that are applied on top of the other requirements on the $\mathscr{E}_{\mathrm{T}}$ and lepton and jet $p_{\mathrm{T}}$ and $\eta$.

There are several contributions to the total standardmodel background for the same-sign dilepton events. One of these contributions comes from events for which the charge of one of the leptons is misreconstructed, for instance in $t \bar{t}$ events with two $W$ bosons decaying into leptons. Second, there are events with one prompt lepton and one nonprompt lepton passing the isolation and identification criteria. Finally, there is an irreducible contribution from standard-model processes with two prompt leptons of the same sign; e.g. $W^{ \pm} W^{ \pm}, W Z, Z Z, t \bar{t}+W$, and $t \bar{t}+Z$. Except for $W^{ \pm} W^{ \pm}$, these processes are also the main contributions to the total background for the trilepton subsample. The event yields for the irreducible component of the background for the same-sign dilepton channel and the total background in the case of the trilepton subsample are taken from the simulation. We obtain from the data the predicted number of background events for the first two contributions to the total background in the same-sign dilepton subsample.

For the same-sign dilepton events with at least one electron, the background is estimated from control samples. We determine the charge misidentification rate for electrons using a double-isolated-electron trigger. We require two isolated electrons with the dielectron invariant mass within $10 \mathrm{GeV}$ of the $Z$-boson mass. We select

TABLE II. Event yields in the single lepton channel. Uncertainties reflect the combined statistical and systematic uncertainties. The prediction for the signal is shown for two different values of $A$ and for a fourth-generation-quark mass $m_{q^{\prime}}=550 \mathrm{GeV}$.

\begin{tabular}{|c|c|c|c|c|c|c|c|}
\hline & $1 b 2 \mathrm{~W}$ & $1 b 3 \mathrm{~W}$ & $1 \mathrm{~b} 4 \mathrm{~W}$ & $2 \mathrm{~b} 1 \mathrm{~W}$ & $2 \mathrm{~b} 2 \mathrm{~W}$ & $2 \mathrm{~b} 3 \mathrm{~W}$ & $2 \mathrm{~b} 4 \mathrm{~W}$ \\
\hline$t \bar{t}+$ jets & $5630 \pm 410$ & $230_{-26}^{+29}$ & $3.0_{-1.3}^{+1.9}$ & $819_{-62}^{+59}$ & $2810 \pm 240$ & $85_{-10}^{+12}$ & $0.6_{-0.5}^{+0.8}$ \\
\hline$W+$ jets & $490 \pm 180$ & $8.0_{-3.0}^{+3.1}$ & $0.3_{-0.3}^{+0.9}$ & $150_{-46}^{+47}$ & $37 \pm 12$ & $1.1_{-0.4}^{+1.0}$ & $0.0_{-0.0}^{+0.8}$ \\
\hline$Z+$ jets & $36_{-6}^{+5}$ & $1.0_{-0.1}^{+0.2}$ & 0 & $7.1_{-0.6}^{+1.0}$ & $2.8_{-0.3}^{+1.0}$ & 0 & 0 \\
\hline Single top & $346 \pm 64$ & $6.5_{-1.5}^{+1.6}$ & $0.2_{-0.2}^{+0.3}$ & $200 \pm 34$ & $110 \pm 19$ & $2.5_{-0.5}^{+0.7}$ & $0.0_{-0.0}^{+0.1}$ \\
\hline VV & $15 \pm 2$ & $0.4_{-0.1}^{+0.3}$ & $0.0_{-0.0}^{+0.1}$ & $15 \pm 2$ & $1.8 \pm 0.3$ & $0.0_{-0.0}^{+0.1}$ & $0.0_{-0.0}^{+0.1}$ \\
\hline$t \bar{t} V$ & $28 \pm 3$ & $3.4 \pm 0.5$ & $0.1 \pm 0.0$ & $0.7 \pm 0.2$ & $15 \pm 5$ & $1.5_{-0.2}^{+0.3}$ & 0 \\
\hline Total background & $6550 \pm 450$ & $249_{-26}^{+29}$ & $3.6_{-1.3}^{+2.1}$ & $1190_{-85}^{+83}$ & $2970 \pm 240$ & $91_{-10}^{+12}$ & $0.6_{-0.5}^{+1.2}$ \\
\hline Observed & 7003 & 242 & 8 & 1357 & 3043 & 91 & 4 \\
\hline Signal $(A=1)$ & $55 \pm 1$ & $12 \pm 1$ & $0.9 \pm 0.2$ & $1.0_{-0.3}^{+0.2}$ & $49 \pm 2$ & $8.1 \pm 0.4$ & $0.5 \pm 0.2$ \\
\hline Signal $(A=0.8)$ & $85 \pm 2$ & $14 \pm 1$ & $1.0 \pm 0.2$ & $69 \pm 3$ & $66 \pm 2$ & $9.2 \pm 0.4$ & $0.5 \pm 0.2$ \\
\hline
\end{tabular}


TABLE IV. The prediction for the total number of background events compared with the number of observed events in the same-sign dilepton and the trilepton subsamples. The numbers of expected signal events are also shown for two possible scenarios.

\begin{tabular}{|c|c|c|c|c|}
\hline Type & 2 muons & 2 electrons & Electron + muon & Trilepton \\
\hline Irreducible background & $0.77 \pm 0.08$ & $0.59 \pm 0.08$ & $1.10 \pm 0.11$ & $0.96 \pm 0.12$ \\
\hline Background from charge misid & $\ldots$ & $0.47 \pm 0.08$ & $0.71 \pm 0.06$ & $\cdots$ \\
\hline Background from fake leptons & $0.06 \pm 0.06$ & $0.30 \pm 0.15$ & $0.46 \pm 0.17$ & $\cdots$ \\
\hline Total background & $0.83 \pm 0.11$ & $1.36 \pm 0.19$ & $2.27 \pm 0.22$ & $0.96 \pm 0.12$ \\
\hline Observed & 2 & 2 & 2 & 1 \\
\hline Signal $\left(A=1, m_{q^{\prime}}=550 \mathrm{GeV}\right)$ & $3.31 \pm 0.15$ & $2.03 \pm 0.36$ & $5.29 \pm 0.19$ & $3.37 \pm 0.16$ \\
\hline Signal $\left(A=0.8, m_{q^{\prime}}=550 \mathrm{GeV}\right)$ & $3.79 \pm 0.15$ & $2.29 \pm 0.36$ & $6.00 \pm 0.19$ & $3.65 \pm 0.16$ \\
\hline
\end{tabular}

events with $\not E_{\mathrm{T}}<20 \mathrm{GeV}$ and a transverse mass $M_{\mathrm{T}}=$ $\sqrt{2 p_{\mathrm{T}}^{\ell} \mathbb{E}_{\mathrm{T}}}\left[1-\cos \left(\Delta \phi\left(\ell, \mathscr{E}_{\mathrm{T}}\right)\right)\right]$ less than $25 \mathrm{GeV}$ to suppress background from top-quark and $W+$ jets events. We define the charge misidentification ratio $R$ as the number of events with two electrons of the same sign divided by twice the number of events with two electrons of opposite sign, i.e. $R=N_{S S} / 2 N_{O S}$. We obtain $0.14 \%$ and $1.4 \%$ for barrel and end-cap electron candidates, respectively. After the full event selection is applied, with the exception of the electron sign requirement, we obtain a number of selected data events with two electrons and with an electron and a muon in the final state. The background with two electrons or with an electron and a muon with the same sign is obtained by taking the number of oppositesign events and scaling it with $R$. The $p_{\mathrm{T}}$ spectrum of the electrons in the control sample and the signal region is similar. Therefore, no correction is applied for the $p_{\mathrm{T}}$ dependency of the charge misidentification ratio.

Another important background contribution to the samesign dilepton channel originates from jets being misidentified as an electron or a muon ("fake" leptons). Two collections of leptons, "loose" and "tight", are defined based on the isolation and identification criteria. Loose leptons are required to fulfill $I_{\text {rel }}<0.2$, in contrast with $I_{\text {rel }}<0.125(0.1)$ for tight muons (electrons). Moreover, we require $|\eta|<2.5$ and $p_{\mathrm{T}}<10(15)$ for loose muons (electrons). Additionally, several identification criteria, intended to ensure the consistency of the lepton track with the primary vertex, are relaxed. We require at least one loose electron or muon. Additionally, we require $\not_{\mathrm{T}}<$ $20 \mathrm{GeV}$ and $M_{T}<25 \mathrm{GeV}$ to suppress background from top-quark and $W+$ jets events. Moreover, we veto events with leptons of the same flavor which have a dilepton mass within $20 \mathrm{GeV}$ of the $Z$-boson mass. We count the number of loose and tight leptons with a $p_{\mathrm{T}}$ below $35 \mathrm{GeV}$. The threshold on the $p_{\mathrm{T}}$ is required to suppress contamination from $W+$ jets events, which would bias the estimation, because leptons produced in jets have typically a soft $p_{\mathrm{T}}$ spectrum. The probability that a loose (L) lepton passes the tight $(\mathrm{T})$ selection criteria is then given by the ratio $\epsilon_{\mathrm{TL}}=N_{\mathrm{T}} / N_{\mathrm{L}}$. To estimate the number of events from the background source with a nonprompt lepton, we count the number of events in data that pass the event selection criteria with one lepton passing the tight selection criteria and a second lepton passing the loose, but not the tight, criteria. This yield is multiplied by $\epsilon_{\mathrm{TL}}\left(1-\epsilon_{\mathrm{TL}}\right)$ to determine the number of events with a nonprompt lepton in the analysis. The statistical uncertainty on the estimated number of events is large because only a few events are selected with one tight and one loose, but not tight, lepton.

The total number of expected background events for the same-sign dilepton and trilepton channels is given in Table IV.

\section{SETTING LOWER LIMITS ON THE FOURTH-GENERATION QUARK MASSES}

We have defined different subsamples according to the reconstructed final state. In each of the different subsamples, we reconstruct observables that are sensitive to the presence of the fourth-generation quarks. These observables are used as input to a fit of the combined distributions for the standard-model (background-only) hypothesis and the signal-plus-background hypothesis. With the profile likelihood ratio as a test statistic, we calculate the $95 \%$ confidence level (CL) upper limits on the combined input cross section of the signal as a function of the $V_{\mathrm{CKM}}^{4 \times 4}$ parameter $A$ and the mass of the fourth-generation quarks.

\section{A. Observables sensitive to the fourth-generation quark production}

The expected number of events is small in the subsamples with two leptons of the same sign, the trilepton subsample, and the two single-lepton subsamples with four $W$-boson candidates. As a consequence, the event counts in each of these subsamples are used as the observable. Table IV summarizes the event counts for the subsamples with two leptons of the same sign and the trilepton subsample.

In the single-lepton subsamples with one or three $W$ bosons, we use $S_{\mathrm{T}}$ as the observable to discriminate between the standard model background and the fourthgeneration signal, where $S_{\mathrm{T}}$ is defined as the scalar sum of the transverse momenta of the reconstructed objects in the final state, namely: 

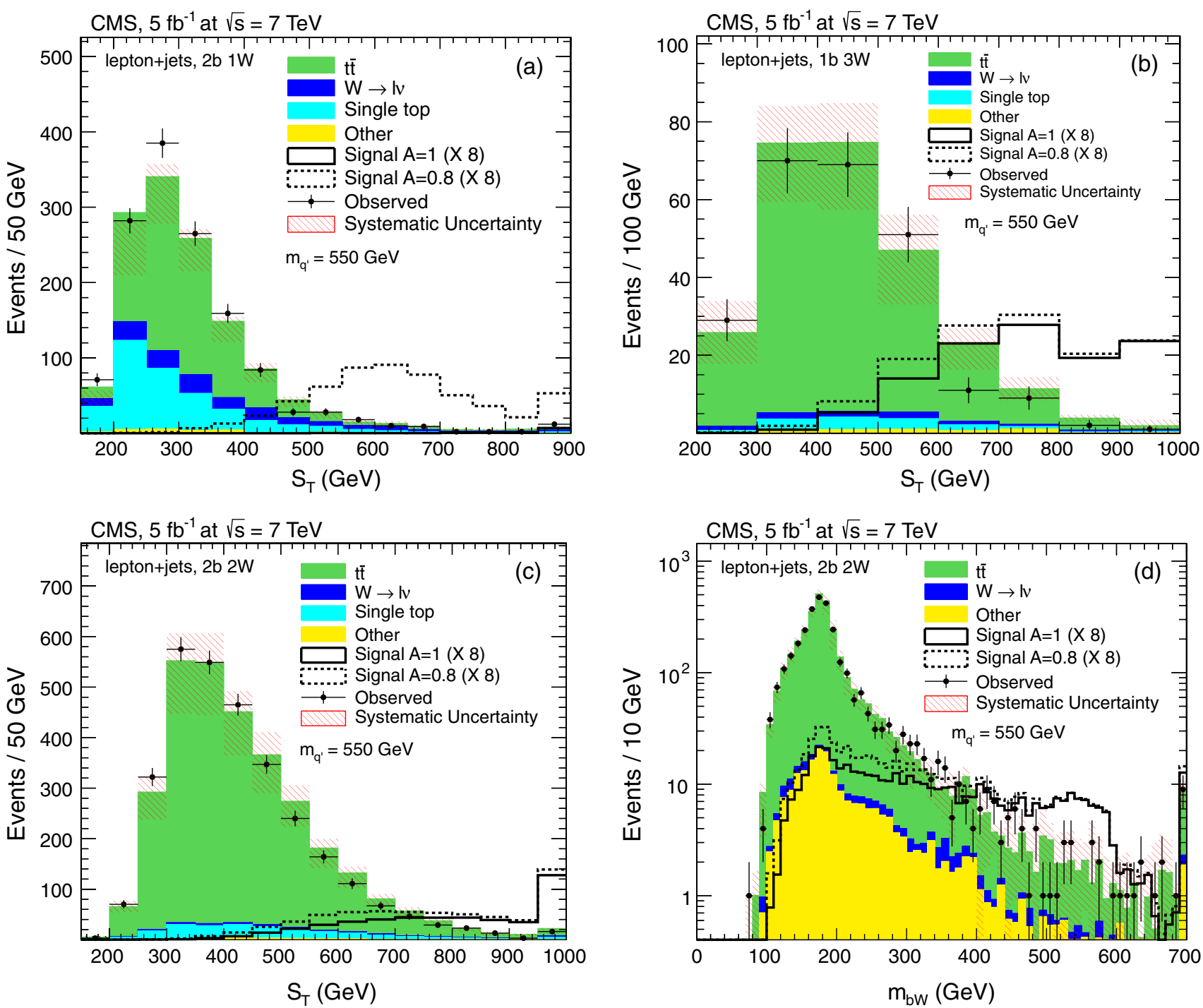

FIG. 1 (color online). The $S_{\mathrm{T}}$ distribution for the subsamples with two $b$ jets and one $W$ boson (a), one $b$ jet and three $W$ bosons (b), two $b$ jets and two $W$ bosons (c), and the $m_{b W}$ distribution for the subsample with two $b$ jets and two $W$ bosons (d). The data distributions of these observables are compared to their expectation from the simulation assuming the fitted nuisance parameters. The fitted values of the nuisance parameters represent the systematic shifts that are applied on the simulation to fit the data in the background-only hypothesis. As an illustration, the total uncertainty band is shown around the simulated expected distribution before taking into account the fitted values of the nuisance parameters. The expected distribution for a signal is shown for two different values of the $V_{\mathrm{CKM}}^{4 \times 4}$ parameter $A$ and for $b^{\prime}$ and $t^{\prime}$ masses of $550 \mathrm{GeV}$. The cross section of the signal in the plots is scaled by a factor of eight for visibility. The last bin in all the histograms includes the overflow. We do not expect much signal for $A=1$ in (a), because the subsample with two $b$ jets and one $W$ boson is mainly sensitive to single $t^{\prime}$-quark production.

$$
S_{\mathrm{T}}=\not E_{\mathrm{T}}+p_{\mathrm{T}}^{l}+p_{\mathrm{T}}^{b}+p_{\mathrm{T}}^{j}+\sum_{i=0}^{N} p_{\mathrm{T}}^{W_{q \bar{q}}^{i}},
$$

where the sum runs over the number of reconstructed hadronically decaying $W$ bosons; $p_{\mathrm{T}}^{l}$ is the $p_{\mathrm{T}}$ of the lepton, $p_{\mathrm{T}}^{b}$ the $p_{\mathrm{T}}$ of the $b$ jet, $p_{\mathrm{T}}^{j}$ the $p_{\mathrm{T}}$ of the second $b$ jet or, if there is no additional jet identified as a $b$ jet, the $p_{\mathrm{T}}$ of the jet with the highest transverse momentum in the event that is not used in the $W$-boson reconstruction, and $p_{\mathrm{T}}^{W_{q \bar{q}}^{i}}$ the $p_{\mathrm{T}}$ of the $i^{\text {th }}$ reconstructed $W$ boson decaying to jets. In general, the decay products of the fourth-generation quarks are expected to have higher transverse momenta compared to the standard-model background. This is shown in Fig. 1 for three of the subsamples. The dominant contribution to the selected signal events in the subsample with two $b$ jets and one $W$ boson would come from the $t^{\prime} b$ process. Almost no signal events are selected for $A=1$, because in that case, the production cross section of $t^{\prime} b$ is equal to zero. The subsamples with two $W$ bosons are dominated by $t \bar{t}$ events. In this case, we use two sensitive observables: $S_{\mathrm{T}}$ and the mass of the hadronic $b W$ system, 
TABLE V. Overview of the observables used in the limit calculation

\begin{tabular}{lc}
\hline \hline Subsample & Observable \\
\hline Single-lepton $1 W$ & $S_{\mathrm{T}}$ \\
Single-lepton $2 W$ & $S_{\mathrm{T}}$ and $m_{b W}$ \\
Single-lepton $3 W$ & $S_{\mathrm{T}}$ \\
Single-lepton $4 W$ & Event yield \\
Same-sign dilepton & Event yield \\
Trilepton & Event yield \\
\hline \hline
\end{tabular}

$m_{b W}$. The latter observable is sensitive to the fourthgeneration physics, because of the higher mass of a hypothetical fourth-generation $t^{\prime}$ quark compared to the top-quark mass. To obtain a higher sensitivity with the $m_{b W}$ observable, four jets need to be assigned to the quarks to reconstruct the final state $t^{\prime} \bar{t}^{\prime} \rightarrow W b W b \rightarrow q \bar{q} b \ell \nu_{\ell} b$. Therefore, six observables with discriminating power between correct and wrong jet/quark assignments are combined with a likelihood ratio method. These observables are angles between the decay products, the $W$-boson mass, the transverse momentum of the top quark decaying to hadrons, and an observable related to the values of the $b$-jet identification variable for the jets. The jet/quark assignment with the largest value of the likelihood ratio is chosen. The mass of the $b W$ system is then reconstructed from this chosen jet/quark assignment. The lower plots in Fig. 1 show the projections of the two-dimensional $S_{\mathrm{T}}$ versus $m_{b W}$ distribution.

An overview of the observables used in the fit for the presence of the fourth-generation quarks is presented in Table V.

\section{B. Fitting for the presence of fourth-generation quarks}

We construct a single histogram "template" that contains the information of the sensitive observables from all the subsamples. Different template distributions are made for the signal corresponding to the different values of $A$ and the fourth-generation quark masses $m_{q^{\prime}}$. The binning of the two-dimensional observable distribution in the singlelepton subsamples with two $W$ bosons is defined using the following procedure. We use a binning in the dimension of $m_{b W}$ such that the top-quark pair background events are uniformly distributed over the bins. Second, the binning in the dimension of $S_{\mathrm{T}}$ in each of the $m_{b W}$ bins is chosen to obtain uniformly distributed top-quark pair events also in this dimension.

The templates of the sensitive observables are used as input to obtain the likelihoods for the background-only and the signal-plus-background hypotheses. Systematic uncertainties are taken into account by introducing nuisance parameters, which may affect the shape and the normalization of the templates. In a case where the systematic uncertainty alters the shape of the templates, template morphing $[43,44]$ is used to interpolate linearly on a bin-by-bin basis between the nominal templates and systematically shifted ones.

The normalization of the templates is affected by the uncertainty in the integrated luminosity, the lepton efficiency, and the normalization of the background processes. The integrated luminosity is measured with a precision of $2.2 \%$ [45] and has the same normalization effect on all the templates. The uncertainty in the lepton efficiency is a combination of the uncertainties in the trigger, selection, and identification efficiencies, which amounts to $3 \%$ and $5 \%$ for muon and electron, respectively. For the uncertainty in the normalization of the background processes, we use the uncertainties in the production cross section of the various standard-model processes. The most important contributions that affect the normalization of the templates are the $12 \%$ [33] (30\%) uncertainty for the top-quark pair (single-top) production cross section and a 50\% uncertainty for the $W$ production cross section because of the large fraction of selected events with jets from heavy-flavor quarks. For the multilepton channel, we take into account the uncertainties in the background estimation obtained from the data. We also include the uncertainties in the production cross sections of $Z$ (5\% [34]), $W W(35 \%)$, $W Z(42 \%), Z Z(27 \%), t \bar{t}+W(19 \%), t \bar{t}+Z(28 \%)$, and $W^{ \pm} W^{ \pm}(49 \%)$. The uncertainties in the normalization of diboson and top-quark pair production in association with a boson are taken from a comparison of the next-to-leadingorder and the LO predictions.

The largest systematic effects on the shape of the templates originate from the jet energy corrections [31] and the scale factors between data and simulation for the $b$-jet efficiency and the probability that a light quark or gluon is identified as a $b$ jet [32]. These effects are estimated by varying the nominal value by \pm 1 standard deviation. The uncertainty in the jet energy resolution of about $10 \%$ has a relatively small effect on the expected limits. The same is true for the uncertainty in the modeling of multiple interactions in the same beam crossing. The latter effect is evaluated by varying the average number of interactions in the simulation by $8 \%$.

The probability density functions of the backgroundonly and the signal-plus-background hypotheses are fitted to the data to fix the nuisance parameters in both models. In the signal-plus-background model, an additional variable, defined as the cross section for the fourth-generation signal obtained by combining the separate search channels, is included. In the combined cross section variable, the relative fraction of each fourth-generation signal process is fixed according to the probed model parameters $\left(A, m_{q^{\prime}}\right)$. Using a Gaussian approximation for the probability density function of the test statistic, we determine the 95\% CL expected and observed limits on the combined cross section variable using the $\mathrm{CL}_{s}$ criterion [46-48]. We exclude the point $\left(A, m_{q^{\prime}}\right)$ at the $95 \% \mathrm{CL}$ if the upper limit on the combined cross section variable is smaller than its 
predicted value within the fourth-generation model. The procedure is repeated for each value of $A$ and $m_{q^{\prime}}$.

\section{Results and discussion}

We use the $\mathrm{CL}_{s}$ procedure to calculate the combined limit for the single-muon, single-electron, same-sign dilepton, and trilepton channels. When the value of the $V_{\mathrm{CKM}}^{4 \times 4}$ parameter $A$ approaches unity, the standard model single-top and the $t^{\prime} b^{\prime}$ processes reach their maximal values for the production cross section. When the value of $A$ decreases, the cross section of these processes decreases linearly with $A$. At the same time, the expected cross section of the $t^{\prime} b$ and $t b^{\prime}$ processes increases with $(1-A)$ and is equal to zero for $A=1$. Therefore, the $t^{\prime} b$ and $t b^{\prime}$ processes are expected to enhance the sensitivity for fourth-generation quarks when the parameter $A$ decreases. This is visible in the upper part of Fig. 2 where both the expected and observed limits on $m_{q^{\prime}}$ are more stringent for smaller values of $A$. For instance, the limit on the fourth-generation quark masses increases by $70 \mathrm{GeV}$ for $A=0.9$ compared to the value of the limit for $A \sim 1$. While the $t^{\prime} b$ and $t b^{\prime}$ processes do not contribute for $A \sim 1$, the inclusion of the $t^{\prime} b^{\prime}$ process results in a more stringent limit (a difference of about $30 \mathrm{GeV}$ ) compared to when this process is not taken into account.

The existence of fourth-generation quarks with degenerate masses is excluded for all parameter values below the line using the assumed model of the $V_{\mathrm{CKM}}^{4 \times 4}$ matrix. In particular, fourth-generation quarks with a degenerate mass below $685 \mathrm{GeV}$ are excluded at the $95 \%$ CL for a parameter value of $A \sim 1$. It is worth noting that no limits can be set for $A$ exactly equal to unity $(A=1)$, because in this special case, the fourth-generation quarks would be stable in the assumed model. The analysis is, however, valid for values of A extremely close to unity. The distance between the primary vertex and the decay vertex of the fourth-generation quarks is less than $1 \mathrm{~mm}$ for $1-A>$ $2 \times 10^{-14}$, a number obtained using the LO formula for the decay width of the top quark in which the top-quark mass is replaced with a fourth-generation-quark mass of $600 \mathrm{GeV}$.

Up to now, the masses of the fourth-generation quarks were assumed to be degenerate. However, if a fourth generation of chiral quarks exists, this is not necessarily the case. Therefore, it is interesting to study how the limit would change for nondegenerate quark masses. If we assume nondegenerate masses, another decay channel for the fourth-generation quarks is possible. Namely, the branching fraction for the decay of $t^{\prime}\left(b^{\prime}\right)$ into $b^{\prime}\left(t^{\prime}\right)$, and an off-shell $W$ boson becomes nonzero. For values of the mass splitting up to about $25 \mathrm{GeV}$, this branching fraction is small as noted in the introduction. We assume a mass splitting of $25 \mathrm{GeV}$ and unchanged branching fractions for the $t^{\prime}$ and $b^{\prime}$ decays. The sensitivity of the analysis increases or decreases depending on the specific values of the masses and hence the production cross sections of
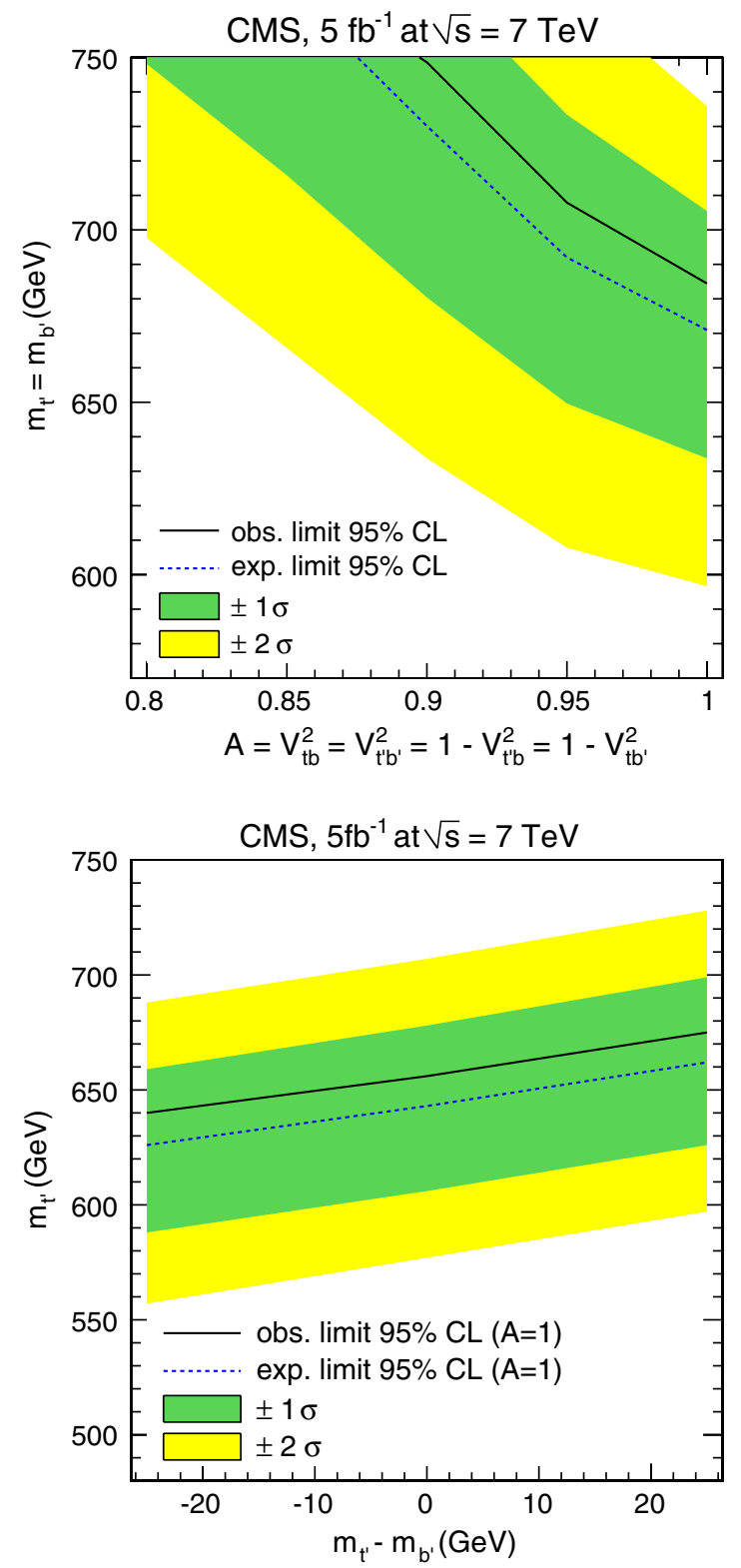

FIG. 2 (color online). Top: Exclusion limit on $m_{t^{\prime}}=m_{b^{\prime}}$ as a function of the $V_{\text {CKM }}^{4 \times 4}$ parameter $A$. The parameter values below the solid line are excluded at 95\% CL. The inner (outer) band indicates the $68 \%$ (95\%) confidence interval around the expected limit. The slope indicates the sensitivity of the analysis to the $t^{\prime} b$ and $t b^{\prime}$ processes. Bottom: For a $V_{\mathrm{CKM}}^{4 \times 4}$ parameter value $A \sim 1$, the exclusion limit on $m_{t^{\prime}}$ versus $m_{t^{\prime}}-m_{b^{\prime}}$ is shown. The exclusion limit is calculated for mass differences up to $25 \mathrm{GeV}$. The existence of up-type fourth-generation quarks with mass values below the observed limit are excluded at the 95\% CL.

the fourth-generation quarks. The effect of the mass difference between the fourth-generation quarks on the exclusion limit is shown in the bottom plot of Fig. 2 for a $V_{\mathrm{CKM}}^{4 \times 4}$ parameter $A \sim 1$. For instance, in case $m_{t^{\prime}}=m_{b^{\prime}}+$ $25 \mathrm{GeV}\left(m_{t^{\prime}}=m_{b^{\prime}}-25 \mathrm{GeV}\right)$, the limit on $m_{t^{\prime}}$ increases about $+20(-20) \mathrm{GeV}$ with respect to the degenerate-mass 
case. To obtain this limit, we do not take into account the electroweak $t^{\prime} b^{\prime}$ process, which results in more conservative exclusion limits. In particular, one observes that quarks with degenerate masses below about $655 \mathrm{GeV}$ are excluded at the $95 \% \mathrm{CL}$ compared to $685 \mathrm{GeV}$ when the $t^{\prime} b^{\prime}$ process is included.

\section{SUMMARY}

Results from a search for a fourth generation of quarks have been presented. A simple model for a unitary CKM matrix has been defined based on a single parameter $A=\left|V_{t b}\right|^{2}=\left|V_{t^{\prime} b^{\prime}}\right|^{2}$. Degenerate masses have been assumed for the fourth-generation quarks, hence $m_{t^{\prime}}=$ $m_{b^{\prime}}$. The information is combined from different subsamples corresponding to different final states with at least one electron or muon. Observables have been constructed in each of the subsamples and used to differentiate between the standard-model background and the processes with fourth-generation quarks. With this strategy, the search for singly and pair-produced $t^{\prime}$ and $b^{\prime}$ quarks has been combined in a coherent way into a single analysis. Model-dependent limits are derived on the mass of the quarks and the $V_{\mathrm{CKM}}^{4 \times 4}$ matrix element $A$. The existence of fourth-generation quarks with masses below $685 \mathrm{GeV}$ is excluded at $95 \%$ confidence level for minimal off-diagonal mixing between the third- and the fourth-generation quarks. A nonzero cross section for the single fourthgeneration quark production processes, corresponding to a value of the $V_{\mathrm{CKM}}^{4 \times 4}$ parameter $A<1$, gives rise to a more stringent limit. When a mass difference of $25 \mathrm{GeV}$ is assumed between $t^{\prime}$ and $b^{\prime}$ quarks, the limit on $m_{t^{\prime}}$ shifts by about $+20(-20) \mathrm{GeV}$ for $m_{t^{\prime}}=m_{b^{\prime}}+25 \mathrm{GeV}\left(m_{t^{\prime}}=\right.$ $m_{b^{\prime}}-25 \mathrm{GeV}$ ). These results significantly reduce the allowed parameter space for a fourth generation of fermions and raise the lower limits on the masses of the fourth generation quarks to the region where nonperturbative effects of the weak interactions are important.

\section{ACKNOWLEDGMENTS}

We congratulate our colleagues in the CERN accelerator departments for the excellent performance of the LHC machine. We thank the technical and administrative staff at CERN and other CMS institutes, and acknowledge support from BMWF and FWF (Austria); FNRS and FWO (Belgium); CNPq, CAPES, FAPERJ, and FAPESP (Brazil); MES (Bulgaria); CERN; CAS, MoST, and NSFC (China); COLCIENCIAS (Colombia); MSES (Croatia); RPF (Cyprus); MoER, SF0690030s09 and ERDF (Estonia); Academy of Finland, MEC, and HIP (Finland); CEA and CNRS/IN2P3 (France); BMBF, DFG, and HGF (Germany); GSRT (Greece); OTKA and NKTH (Hungary); DAE and DST (India); IPM (Iran); SFI (Ireland); INFN (Italy); NRF and WCU (Korea); LAS (Lithuania); CINVESTAV, CONACYT, SEP, and UASLP-FAI (Mexico); MSI (New Zealand); PAEC (Pakistan); MSHE and NSC (Poland); FCT (Portugal); JINR (Armenia, Belarus, Georgia, Ukraine, Uzbekistan); MON, RosAtom, RAS and RFBR (Russia); MSTD (Serbia); SEIDI and CPAN (Spain); Swiss Funding Agencies (Switzerland); NSC (Taipei); ThEP, IPST and NECTEC (Thailand); TUBITAK and TAEK (Turkey); NASU (Ukraine); STFC (United Kingdom); DOE and NSF (USA). Individuals have received support from the Marie-Curie program and the European Research Council (European Union); the Leventis Foundation; the A.P. Sloan Foundation; the Alexander von Humboldt Foundation; the Austrian Science Fund (FWF); the Belgian Federal Science Policy Office; the Fonds pour la Formation à la Recherche dans l'Industrie et dans l'Agriculture (FRIA-Belgium); the Agentschap voor Innovatie door Wetenschap en Technologie (IWTBelgium); the Ministry of Education, Youth and Sports (MEYS) of Czech Republic; the Council of Science and Industrial Research, India; the Compagnia di San Paolo (Torino); and the HOMING PLUS program of Foundation for Polish Science, cofinanced from European Union, Regional Development Fund.
[1] D. E. Groom et al. (Particle Data Group), Eur. Phys. J. C 15, 357 (2000).

[2] H. Flaecher, M. Goebel, J. Haller, A. Hoecker, K. Moenig, and J. Stelzer, Eur. Phys. J. C 60, 543 (2009).

[3] M. Buchkremer, J.-M. Gerard, and F. Maltoni, J. High Energy Phys. 06 (2012) 135.

[4] N. Cabibbo, Phys. Rev. Lett. 10, 531 (1963).

[5] M. Kobayashi and T. Maskawa, Prog. Theor. Phys. 49, 652 (1973).

[6] B. Pontecorvo, Sov. Phys. JETP 6, 429 (1957).

[7] Z. Maki, M. Nakagawa, and S. Sakata, Prog. Theor. Phys. 28, 870 (1962).
[8] E. Asilar, E. Cavlan, O. Dogangun, S. Kefeli, E. Ozcan, M. Sahin, and G. Unel, Eur. Phys. J. C 72, 1966 (2012).

[9] G. Aad et al. (ATLAS), Phys. Rev. Lett. 109, 032001 (2012).

[10] G. Aad et al. (ATLAS), Phys. Rev. D 86, 012007 (2012).

[11] G. Aad et al. (ATLAS), J. High Energy Phys. 04 (2012) 069.

[12] G. Aad et al. (ATLAS), Phys. Rev. Lett. 108, 261802 (2012).

[13] S. Chatrchyan et al. (CMS), Phys. Lett. B 716, 103 (2012).

[14] S. Chatrchyan et al. (CMS), J. High Energy Phys. 05 (2012) 123

[15] S. Chatrchyan et al. (CMS), Phys. Lett. B 718, 307 (2012). 
[16] M. S. Chanowitz, M. A. Furman, and I. Hinchliffe, Nucl. Phys. B153, 402 (1979).

[17] K. Nakamura et al. (Particle Data Group), Phys. Rev. D 86, 010001 (2012).

[18] V. M. Abazov et al. (D0), Phys. Rev. D 85, 091104 (2012).

[19] Y. Chao, K.-F. Chen, S.-K. Chen, W.-S. Hou, B.-Y. Huang, and Y.-J. Lei, Phys. Rev. D 84, 014029 (2011).

[20] S. Chatrchyan et al. (CMS), JINST 3, S08004 (2008).

[21] CMS Collaboration (CMS), Commissioning of the Particle-Flow Reconstruction in Minimum-Bias and Jet Events from Collisions at $7 \mathrm{TeV}$, CMS Physics Analysis Summary CMS-PAS-PFT-10-002 (2010), http://cdsweb .cern.ch/record/1279341.

[22] M. Cacciari, G. P. Salam, and G. Soyez, J. High Energy Phys. 04 (2008) 063.

[23] S. Alioli, P. Nason, C. Oleari, and E. Re, J. High Energy Phys. 09 (2009) 111.

[24] E. Re, Eur. Phys. J. C 71, 1547 (2011).

[25] T. Sjöstrand, S. Mrenna, and P.Z. Skands, J. High Energy Phys. 05 (2006) 026.

[26] J. Alwall, M. Herquet, F. Maltoni, O. Mattelaer, and T. Stelzer, J. High Energy Phys. 06 (2011) 128.

[27] R. Field, arXiv:1010.3558.

[28] M. L. Mangano, M. Moretti, F. Piccinini, and M. Treccani, J. High Energy Phys. 01 (2007) 013.

[29] J. Pumplin, D. R. Stump, J. Huston, H. L. Lai, P. Nadolsky, and W. K. Tung, J. High Energy Phys. 07 (2002) 012.

[30] J. Allison et al., IEEE Trans. Nucl. Sci. 53, 270 (2006).

[31] S. Chatrchyan et al. (CMS), JINST 6, P11002 (2011).

[32] CMS Collaboration (CMS), b-Jet Identification in the CMS Experiment, CMS Physics Analysis Summary CMS-PAS-BTV-11-004 (2011), http://cdsweb.cern.ch/ record/1427247.
[33] S. Chatrchyan et al. (CMS), Phys. Rev. D 84, 092004 (2011).

[34] S. Chatrchyan et al. (CMS), J. High Energy Phys. 10 (2011) 132.

[35] N. Kidonakis, Phys. Rev. D 83, 091503 (2011).

[36] N. Kidonakis, Phys. Rev. D 81, 054028 (2010).

[37] N. Kidonakis, Phys. Rev. D 82, 054018 (2010).

[38] V. Hirschi, R. Frederix, S. Frixione, M. V. Garzelli, F. Maltoni, and R. Pittau, J. High Energy Phys. 05 (2011) 044.

[39] J. M. Campbell and R. K. Ellis, Nucl. Phys. B, Proc. Suppl. 205-206, 10 (2010).

[40] J. M. Campbell and R. K. Ellis, Phys. Rev. D 60, 113006 (1999).

[41] M. Aliev, H. Lacker, U. Langenfeld, S. Moch, P. Uwer, and M. Wiedermann, Comput. Phys. Commun. 182, 1034 (2011).

[42] J. M. Campbell, R. Frederix, F. Maltoni, and F. Tramontano, J. High Energy Phys. 10 (2009) 042.

[43] A. L. Read, Nucl. Instrum. Methods Phys. Res., Sect. A 425, 357 (1999).

[44] J. S. Conway, in Proceedings of PHYSTAT 2011 Workshop on Statistical Issues Related to Discovery Claims in Search Experiments and Unfolding, edited by H.B. Propser and L. Lyons (CERN, 2001), p. 115 [http:// cdsweb.cern.ch/record/1306523].

[45] CMS Collaboration, CMS Physics Analysis Summary Report No. CMS-PAS-SMP-12-008, 2012 [http://cdsweb .cern.ch/record/1434360].

[46] G. Cowan, K. Cranmer, E. Gross, and O. Vitells, Eur. Phys. J. C 71, 1554 (2011).

[47] T. Junk, Nucl. Instrum. Methods Phys. Res., Sect. A 434, 435 (1999).

[48] A. L. Read, J. Phys. G 28, 2693 (2002).

S. Chatrchyan, ${ }^{1}$ V. Khachatryan, ${ }^{1}$ A. M. Sirunyan, ${ }^{1}$ A. Tumasyan, ${ }^{1}$ W. Adam, ${ }^{2}$ E. Aguilo, ${ }^{2}$ T. Bergauer, ${ }^{2}$ M. Dragicevic, ${ }^{2}$ J. Erö, ${ }^{2}$ C. Fabjan, ${ }^{2, b}$ M. Friedl, ${ }^{2}$ R. Frühwirth, ${ }^{2, b}$ V. M. Ghete, ${ }^{2}$ J. Hammer, ${ }^{2}$ N. Hörmann, ${ }^{2}$ J. Hrubec, ${ }^{2}$ M. Jeitler, ${ }^{2, b}$ W. Kiesenhofer, ${ }^{2}$ V. Knünz, ${ }^{2}$ M. Krammer, ${ }^{2, b}$ I. Krätschmer, ${ }^{2}$ D. Liko, ${ }^{2}$ I. Mikulec, ${ }^{2}$

M. Pernicka, ${ }^{2, a}$ B. Rahbaran, ${ }^{2}$ C. Rohringer, ${ }^{2}$ H. Rohringer, ${ }^{2}$ R. Schöfbeck, ${ }^{2}$ J. Strauss, ${ }^{2}$ A. Taurok, ${ }^{2}$

W. Waltenberger, ${ }^{2}$ G. Walzel, ${ }^{2}$ E. Widl, ${ }^{2}$ C.-E. Wulz,${ }^{2, b}$ V. Mossolov, ${ }^{3}$ N. Shumeiko, ${ }^{3}$ J. Suarez Gonzalez, ${ }^{3}$ M. Bansal, ${ }^{4}$ S. Bansal, ${ }^{4}$ T. Cornelis, ${ }^{4}$ E. A. De Wolf, ${ }^{4}$ X. Janssen, ${ }^{4}$ S. Luyckx,${ }^{4}$ L. Mucibello, ${ }^{4}$ S. Ochesanu, ${ }^{4}$ B. Roland,${ }^{4}$ R. Rougny, ${ }^{4}$ M. Selvaggi, ${ }^{4}$ Z. Staykova, ${ }^{4}$ H. Van Haevermaet,${ }^{4}$ P. Van Mechelen, ${ }^{4}$ N. Van Remortel, ${ }^{4}$ A. Van Spilbeeck, ${ }^{4}$ F. Blekman, ${ }^{5}$ S. Blyweert, ${ }^{5}$ J. D'Hondt, ${ }^{5}$ R. Gonzalez Suarez,${ }^{5}$ A. Kalogeropoulos, ${ }^{5}$ M. Maes, ${ }^{5}$ A. Olbrechts, ${ }^{5}$ W. Van Doninck,${ }^{5}$ P. Van Mulders, ${ }^{5}$ G. P. Van Onsem, ${ }^{5}$ I. Villella, ${ }^{5}$ B. Clerbaux,${ }^{6}$ G. De Lentdecker, ${ }^{6}$ V. Dero, ${ }^{6}$ A. P. R. Gay, ${ }^{6}$ T. Hreus, ${ }^{6}$ A. Léonard, ${ }^{6}$ P.E. Marage, ${ }^{6}$ A. Mohammadi, ${ }^{6}$ T. Reis, ${ }^{6}$ L. Thomas, ${ }^{6}$ G. Vander Marcken, ${ }^{6}$ C. Vander Velde, ${ }^{6}$ P. Vanlaer, ${ }^{6}$ J. Wang, ${ }^{6}$ V. Adler, ${ }^{7}$ K. Beernaert, ${ }^{7}$ A. Cimmino, ${ }^{7}$ S. Costantini, ${ }^{7}$ G. Garcia, ${ }^{7}$ M. Grunewald, ${ }^{7}$ B. Klein, ${ }^{7}$ J. Lellouch, ${ }^{7}$ A. Marinov, ${ }^{7}$ J. Mccartin, ${ }^{7}$ A. A. Ocampo Rios, ${ }^{7}$ D. Ryckbosch, ${ }^{7}$ N. Strobbe, ${ }^{7}$ F. Thyssen, ${ }^{7}$ M. Tytgat, ${ }^{7}$ P. Verwilligen, ${ }^{7}$ S. Walsh, ${ }^{7}$ E. Yazgan, ${ }^{7}$ N. Zaganidis, ${ }^{7}$ S. Basegmez, ${ }^{8}$ G. Bruno, ${ }^{8}$ R. Castello, ${ }^{8}$ L. Ceard, ${ }^{8}$ C. Delaere, ${ }^{8}$ T. du Pree,${ }^{8}$ D. Favart,${ }^{8}$ L. Forthomme, ${ }^{8}$ A. Giammanco,${ }^{8, \mathrm{c}}$ J. Hollar, ${ }^{8}$ V. Lemaitre, ${ }^{8}$ J. Liao, ${ }^{8}$ O. Militaru, ${ }^{8}$ C. Nuttens, ${ }^{8}$ D. Pagano, ${ }^{8}$ A. Pin,${ }^{8}$ K. Piotrzkowski,${ }^{8}$ N. Schul,${ }^{8}$

J. M. Vizan Garcia, ${ }^{8}$ N. Beliy, ${ }^{9}$ T. Caebergs,${ }^{9}$ E. Daubie, ${ }^{9}$ G. H. Hammad, ${ }^{9}$ G. A. Alves, ${ }^{10}$ M. Correa Martins Junior, ${ }^{10}$ D. De Jesus Damiao, ${ }^{10}$ T. Martins,${ }^{10}$ M.E. Pol, ${ }^{10}$ M. H. G. Souza, ${ }^{10}$ W. L. Aldá Júnior,${ }^{11}$ W. Carvalho, ${ }^{11}$

A. Custódio, ${ }^{11}$ E. M. Da Costa, ${ }^{11}$ C. De Oliveira Martins, ${ }^{11}$ S. Fonseca De Souza,${ }^{11}$ D. Matos Figueiredo,,${ }^{11}$ L. Mundim,${ }^{11}$ H. Nogima, ${ }^{11}$ V. Oguri, ${ }^{11}$ W. L. Prado Da Silva, ${ }^{11}$ A. Santoro, ${ }^{11}$ L. Soares Jorge, ${ }^{11}$ A. Sznajder, ${ }^{11}$ T. S. Anjos, ${ }^{12, \mathrm{~d}}$ C. A. Bernardes, ${ }^{12, \mathrm{~d}}$ F. A. Dias, ${ }^{12, \mathrm{e}}$ T.R. Fernandez Perez Tomei, ${ }^{12}$ E. M. Gregores, ${ }^{12, \mathrm{~d}}$ C. Lagana, ${ }^{12}$ 
F. Marinho, ${ }^{12}$ P. G. Mercadante, ${ }^{12, \mathrm{~d}}$ S. F. Novaes,${ }^{12}$ Sandra S. Padula, ${ }^{12}$ V. Genchev, ${ }^{13, \mathrm{f}}$ P. Iaydjiev, ${ }^{13, \mathrm{f}}$ S. Piperov, ${ }^{13}$ M. Rodozov, ${ }^{13}$ S. Stoykova, ${ }^{13}$ G. Sultanov,${ }^{13}$ V. Tcholakov,${ }^{13}$ R. Trayanov,${ }^{13}$ M. Vutova, ${ }^{13}$ A. Dimitrov,${ }^{14}$ R. Hadjiiska, ${ }^{14}$ V. Kozhuharov, ${ }^{14}$ L. Litov,${ }^{14}$ B. Pavlov, ${ }^{14}$ P. Petkov, ${ }^{14}$ J. G. Bian, ${ }^{15}$ G. M. Chen, ${ }^{15}$ H. S. Chen, ${ }^{15}$ C. H. Jiang, ${ }^{15}$ D. Liang, ${ }^{15}$ S. Liang, ${ }^{15}$ X. Meng, ${ }^{15}$ J. Tao, ${ }^{15}$ J. Wang,,${ }^{15}$ X. Wang, ${ }^{15}$ Z. Wang,,${ }^{15}$ H. Xiao, ${ }^{15}$ M. Xu, ${ }^{15}$ J. Zang, ${ }^{15}$ Z. Zhang, ${ }^{15}$ C. Asawatangtrakuldee, ${ }^{16}$ Y. Ban, ${ }^{16}$ S. Guo, ${ }^{16}$ Y. Guo, ${ }^{16} \mathrm{~W} . \mathrm{Li},{ }^{16} \mathrm{~S} . \mathrm{Liu},{ }^{16}$ Y. Mao, ${ }^{16}$ S. J. Qian, ${ }^{16}$ H. Teng, ${ }^{16}$ D. Wang, ${ }^{16}$ L. Zhang, ${ }^{16}$ B. Zhu, ${ }^{16}$ W. Zou, ${ }^{16}$ C. Avila, ${ }^{17}$ J. P. Gomez, ${ }^{17}$ B. Gomez Moreno, ${ }^{17}$ A. F. Osorio Oliveros, ${ }^{17}$ J. C. Sanabria,${ }^{17}$ N. Godinovic, ${ }^{18}$ D. Lelas, ${ }^{18}$ R. Plestina, ${ }^{18, g}$ D. Polic, ${ }^{18}$ I. Puljak, ${ }^{18, f}$ Z. Antunovic, ${ }^{19}$ M. Kovac, ${ }^{19}$ V. Brigljevic, ${ }^{20}$ S. Duric, ${ }^{20}$ K. Kadija, ${ }^{20}$ J. Luetic,${ }^{20}$ S. Morovic,${ }^{20}$ A. Attikis, ${ }^{21}$ M. Galanti, ${ }^{21}$ G. Mavromanolakis, ${ }^{21}$ J. Mousa, ${ }^{21}$ C. Nicolaou, ${ }^{21}$ F. Ptochos,${ }^{21}$ P. A. Razis, ${ }^{21}$ M. Finger, ${ }^{22}$ M. Finger, Jr., ${ }^{22}$ Y. Assran, ${ }^{23, \mathrm{~h}}$ S. Elgammal, ${ }^{23, \mathrm{i}}$ A. Ellithi Kamel, ${ }^{23, \mathrm{j}} \mathrm{S}$. Khalil, ${ }^{23, \mathrm{i}}$ M. A. Mahmoud, ${ }^{23, \mathrm{k}}$ A. Radi, ${ }^{23,1, \mathrm{~m}}$ M. Kadastik, ${ }^{24}$ M. Müntel,,${ }^{24}$ M. Raidal,${ }^{24}$ L. Rebane, ${ }^{24}$ A. Tiko, ${ }^{24}$ P. Eerola ${ }^{25}$ G. Fedi, ${ }^{25}$ M. Voutilainen,${ }^{25}$ J. Härkönen, ${ }^{26}$ A. Heikkinen, ${ }^{26}$ V. Karimäki ${ }^{26}$ R. Kinnunen,${ }^{26}$ M. J. Kortelainen,${ }^{26}$ T. Lampén, ${ }^{26}$ K. Lassila-Perini, ${ }^{26}$ S. Lehti, ${ }^{26}$ T. Lindén, ${ }^{26}$ P. Luukka, ${ }^{26}$ T. Mäenpää, ${ }^{26}$ T. Peltola, ${ }^{26}$ E. Tuominen, ${ }^{26}$ J. Tuominiemi, ${ }^{26}$ E. Tuovinen, ${ }^{26}$

D. Ungaro, ${ }^{26}$ L. Wendland, ${ }^{26}$ K. Banzuzi, ${ }^{27}$ A. Karjalainen, ${ }^{27}$ A. Korpela, ${ }^{27}$ T. Tuuva, ${ }^{27}$ M. Besancon, ${ }^{28}$ S. Choudhury, ${ }^{28}$ M. Dejardin, ${ }^{28}$ D. Denegri, ${ }^{28}$ B. Fabbro, ${ }^{28}$ J. L. Faure, ${ }^{28}$ F. Ferri, ${ }^{28}$ S. Ganjour,${ }^{28}$ A. Givernaud, ${ }^{28}$ P. Gras, ${ }^{28}$ G. Hamel de Monchenault, ${ }^{28}$ P. Jarry, ${ }^{28}$ E. Locci, ${ }^{28}$ J. Malcles, ${ }^{28}$ L. Millischer, ${ }^{28}$ A. Nayak,${ }^{28}$ J. Rander ${ }^{28}$ A. Rosowsky, ${ }^{28}$ I. Shreyber ${ }^{28}$ M. Titov ${ }^{28}$ S. Baffioni, ${ }^{29}$ F. Beaudette, ${ }^{29}$ L. Benhabib ${ }^{29}$ L. Bianchini, ${ }^{29}$ M. Bluj ${ }^{29, n}$ C. Broutin, ${ }^{29}$ P. Busson, ${ }^{29}$ C. Charlot, ${ }^{29}$ N. Daci, ${ }^{29}$ T. Dahms,${ }^{29}$ L. Dobrzynski ${ }^{29}$ R. Granier de Cassagnac, ${ }^{29}$ M. Haguenauer, ${ }^{29}$ P. Miné ${ }^{29}$ C. Mironov, ${ }^{29}$ I. N. Naranjo, ${ }^{29}$ M. Nguyen,${ }^{29}$ C. Ochando, ${ }^{29}$ P. Paganini, ${ }^{29}$ D. Sabes ${ }^{29}$ R. Salerno, ${ }^{29}$ Y. Sirois, ${ }^{29}$ C. Veelken, ${ }^{29}$ A. Zabi, ${ }^{29}$ J.-L. Agram,${ }^{30, o}$ J. Andrea,${ }^{30}$ D. Bloch,${ }^{30}$ D. Bodin,${ }^{30}$ J.-M. Brom,${ }^{30}$ M. Cardaci,${ }^{30}$ E. C. Chabert, ${ }^{30}$ C. Collard, ${ }^{30}$ E. Conte, ${ }^{30, o}$ F. Drouhin,${ }^{30, o}$ C. Ferro,${ }^{30}$ J.-C. Fontaine,${ }^{30, o}$ D. Gelé, ${ }^{30}$ U. Goerlach, ${ }^{30}$ P. Juillot, ${ }^{30}$ A.-C. Le Bihan, ${ }^{30}$ P. Van Hove,${ }^{30}$ F. Fassi, ${ }^{31}$ D. Mercier ${ }^{31}$ S. Beauceron, ${ }^{32}$ N. Beaupere, ${ }^{32}$ O. Bondu, ${ }^{32}$ G. Boudoul, ${ }^{32}$ J. Chasserat, ${ }^{32}$ R. Chierici, ${ }^{32, f}$ D. Contardo,${ }^{32}$ P. Depasse, ${ }^{32}$ H. El Mamouni, ${ }^{32}$ J. Fay, ${ }^{32}$ S. Gascon, ${ }^{32}$ M. Gouzevitch, ${ }^{32}$ B. Ille, ${ }^{32}$ T. Kurca ${ }^{32}$ M. Lethuillier, ${ }^{32}$ L. Mirabito, ${ }^{32}$ S. Perries, ${ }^{32}$ V. Sordini, ${ }^{32}$ Y. Tschudi, ${ }^{32}$ P. Verdier, ${ }^{32}$ S. Viret, ${ }^{32}$ Z. Tsamalaidze, ${ }^{33, p}$ G. Anagnostou, ${ }^{34}$ S. Beranek,${ }^{34}$ M. Edelhoff, ${ }^{34}$ L. Feld,${ }^{34}$ N. Heracleous,${ }^{34}$ O. Hindrichs, ${ }^{34}$ R. Jussen, ${ }^{34}$ K. Klein, ${ }^{34}$ J. Merz, ${ }^{34}$ A. Ostapchuk,${ }^{34}$ A. Perieanu, ${ }^{34}$ F. Raupach, ${ }^{34}$ J. Sammet, ${ }^{34}$ S. Schael, ${ }^{34}$ D. Sprenger, ${ }^{34}$ H. Weber, ${ }^{34}$ B. Wittmer, ${ }^{34}$ V. Zhukov, ${ }^{34, q}$ M. Ata, ${ }^{35}$ J. Caudron, ${ }^{35}$ E. Dietz-Laursonn, ${ }^{35}$ D. Duchardt,${ }^{35}$ M. Erdmann, ${ }^{35}$ R. Fischer, ${ }^{35}$ A. Güth, ${ }^{35}$ T. Hebbeker,${ }^{35}$ C. Heidemann,${ }^{35}$ K. Hoepfner, ${ }^{35}$ D. Klingebiel,,${ }^{35}$ P. Kreuzer, ${ }^{35}$ C. Magass,${ }^{35}$ M. Merschmeyer, ${ }^{35}$ A. Meyer, ${ }^{35}$ M. Olschewski, ${ }^{35}$ P. Papacz,${ }^{35}$ H. Pieta, ${ }^{35}$ H. Reithler, ${ }^{35}$ S. A. Schmitz, ${ }^{35}$ L. Sonnenschein, ${ }^{35}$ J. Steggemann, ${ }^{35}$

D. Teyssier, ${ }^{35}$ M. Weber, ${ }^{35}$ M. Bontenackels, ${ }^{36}$ V. Cherepanov, ${ }^{36}$ Y. Erdogan, ${ }^{36}$ G. Flügge, ${ }^{36}$ H. Geenen, ${ }^{36}$

M. Geisler, ${ }^{36}$ W. Haj Ahmad ${ }^{36}$ F. Hoehle, ${ }^{36}$ B. Kargoll, ${ }^{36}$ T. Kress, ${ }^{36}$ Y. Kuessel, ${ }^{36}$ A. Nowack, ${ }^{36}$ L. Perchalla, ${ }^{36}$ O. Pooth, ${ }^{36}$ P. Sauerland, ${ }^{36}$ A. Stahl, ${ }^{36}$ M. Aldaya Martin, ${ }^{37}$ J. Behr, ${ }^{37}$ W. Behrenhoff, ${ }^{37}$ U. Behrens, ${ }^{37}$

M. Bergholz, ${ }^{37, \mathrm{r}}$ A. Bethani, ${ }^{37}$ K. Borras,${ }^{37}$ A. Burgmeier, ${ }^{37}$ A. Cakir, ${ }^{37}$ L. Calligaris, ${ }^{37}$ A. Campbell, ${ }^{37}$ E. Castro, ${ }^{37}$ F. Costanza,${ }^{37}$ D. Dammann,${ }^{37}$ C. Diez Pardos,${ }^{37}$ G. Eckerlin, ${ }^{37}$ D. Eckstein,${ }^{37}$ G. Flucke, ${ }^{37}$ A. Geiser,,${ }^{37}$ I. Glushkov, ${ }^{37}$ P. Gunnellini, ${ }^{37}$ S. Habib,${ }^{37}$ J. Hauk,${ }^{37}$ G. Hellwig, ${ }^{37}$ H. Jung, ${ }^{37}$ M. Kasemann, ${ }^{37}$ P. Katsas,${ }^{37}$ C. Kleinwort, ${ }^{37}$ H. Kluge, ${ }^{37}$ A. Knutsson, ${ }^{37}$ M. Krämer, ${ }^{37}$ D. Krücker, ${ }^{37}$ E. Kuznetsova, ${ }^{37}$ W. Lange, ${ }^{37}$ W. Lohmann, ${ }^{37, r}$ B. Lutz, ${ }^{37}$ R. Mankel, ${ }^{37}$ I. Marfin, ${ }^{37}$ M. Marienfeld, ${ }^{37}$ I.-A. Melzer-Pellmann, ${ }^{37}$ A. B. Meyer, ${ }^{37}$ J. Mnich, ${ }^{37}$ A. Mussgiller, ${ }^{37}$ S. Naumann-Emme, ${ }^{37}$ J. Olzem, ${ }^{37}$ H. Perrey,${ }^{37}$ A. Petrukhin, ${ }^{37}$ D. Pitzl, ${ }^{37}$ A. Raspereza, ${ }^{37}$ P. M. Ribeiro Cipriano, ${ }^{37}$ C. Riedl, ${ }^{37}$ E. Ron, ${ }^{37}$ M. Rosin, ${ }^{37}$ J. Salfeld-Nebgen, ${ }^{37}$ R. Schmidt, ${ }^{37, r}$ T. Schoerner-Sadenius, ${ }^{37}$ N. Sen, ${ }^{37}$ A. Spiridonov, ${ }^{37}$ M. Stein,${ }^{37}$ R. Walsh, ${ }^{37}$ C. Wissing, ${ }^{37}$ C. Autermann, ${ }^{38}$ V. Blobel,${ }^{38}$ J. Draeger, ${ }^{38}$ H. Enderle, ${ }^{38}$ J. Erfle,${ }^{38}$ U. Gebbert,${ }^{38}$ M. Görner, ${ }^{38}$ T. Hermanns, ${ }^{38}$ R. S. Höing, ${ }^{38}$ K. Kaschube, ${ }^{38}$ G. Kaussen, ${ }^{38}$ H. Kirschenmann, ${ }^{38}$ R. Klanner,${ }^{38}$ J. Lange, ${ }^{38}$ B. Mura, ${ }^{38}$ F. Nowak,${ }^{38}$ T. Peiffer, ${ }^{38}$ N. Pietsch, ${ }^{38}$ D. Rathjens, ${ }^{38}$ C. Sander,${ }^{38}$ H. Schettler, ${ }^{38}$ P. Schleper, ${ }^{38}$ E. Schlieckau, ${ }^{38}$ A. Schmidt,${ }^{38}$ M. Schröder,${ }^{38}$ T. Schum, ${ }^{38}$ M. Seidel, ${ }^{38}$ V. Sola ${ }^{38}$ H. Stadie, ${ }^{38}$ G. Steinbrück, ${ }^{38}$ J. Thomsen, ${ }^{38}$ L. Vanelderen, ${ }^{38}$ C. Barth, ${ }^{39}$ J. Berger, ${ }^{39}$ C. Böser, ${ }^{39}$ T. Chwalek, ${ }^{39}$ W. De Boer, ${ }^{39}$ A. Descroix, ${ }^{39}$ A. Dierlamm, ${ }^{39}$ M. Feindt, ${ }^{39}$ M. Guthoff,,${ }^{39, f}$ C. Hackstein, ${ }^{39}$ F. Hartmann, ${ }^{39}$ T. Hauth, ${ }^{39, f}$ M. Heinrich, ${ }^{39}$ H. Held ${ }^{39}$ K. H. Hoffmann, ${ }^{39}$ S. Honc,${ }^{39}$ I. Katkov, ${ }^{39, q}$ J. R. Komaragiri, ${ }^{39}$ P. Lobelle Pardo,${ }^{39}$ D. Martschei, ${ }^{39}$ S. Mueller, ${ }^{39}$ Th. Müller, ${ }^{39}$ M. Niegel, ${ }^{39}$ A. Nürnberg, ${ }^{39}$ O. Oberst, ${ }^{39}$ A. Oehler, ${ }^{39}$ J. Ott,${ }^{39}$ G. Quast,${ }^{39}$ K. Rabbertz, ${ }^{39}$ F. Ratnikov, ${ }^{39}$ N. Ratnikova ${ }^{39}$ S. Röcker, ${ }^{39}$ A. Scheurer ${ }^{39}$ F.-P. Schilling, ${ }^{39}$ G. Schott, ${ }^{39}$ H. J. Simonis, ${ }^{39}$ F. M. Stober, ${ }^{39}$ D. Troendle, ${ }^{39}$ R. Ulrich, ${ }^{39}$ J. Wagner-Kuhr, ${ }^{39}$ S. Wayand, ${ }^{39}$ T. Weiler, ${ }^{39}$ M. Zeise, ${ }^{39}$ G. Daskalakis, ${ }^{40}$ T. Geralis, ${ }^{40}$ S. Kesisoglou, ${ }^{40}$ 
A. Kyriakis,${ }^{40}$ D. Loukas, ${ }^{40}$ I. Manolakos,${ }^{40}$ A. Markou, ${ }^{40}$ C. Markou, ${ }^{40}$ C. Mavrommatis, ${ }^{40}$ E. Ntomari, ${ }^{40}$ L. Gouskos, ${ }^{41}$ T. J. Mertzimekis, ${ }^{41}$ A. Panagiotou, ${ }^{41}$ N. Saoulidou ${ }^{41}$ I. Evangelou, ${ }^{42}$ C. Foudas, ${ }^{42}$ P. Kokkas,${ }^{42}$ N. Manthos, ${ }^{42}$ I. Papadopoulos, ${ }^{42}$ V. Patras,${ }^{42}$ G. Bencze, ${ }^{43}$ C. Hajdu ${ }^{43}$ P. Hidas,${ }^{43}$ D. Horvath,${ }^{43, s}$ F. Sikler, ${ }^{43}$ V. Veszpremi, ${ }^{43}$ G. Vesztergombi, ${ }^{43, t}$ N. Beni, ${ }^{44}$ S. Czellar, ${ }^{44}$ J. Molnar, ${ }^{44}$ J. Palinkas, ${ }^{44}$ Z. Szillasi, ${ }^{44}$ J. Karancsi, ${ }^{45}$ P. Raics, ${ }^{45}$ Z. L. Trocsanyi, ${ }^{45}$ B. Ujvari, ${ }^{45}$ S. B. Beri,${ }^{46}$ V. Bhatnagar, ${ }^{46}$ N. Dhingra,${ }^{46}$ R. Gupta, ${ }^{46}$ M. Kaur, ${ }^{46}$ M. Z. Mehta, ${ }^{46}$ N. Nishu, ${ }^{46}$ L. K. Saini, ${ }^{46}$ A. Sharma, ${ }^{46}$ J. B. Singh, ${ }^{46}$ Ashok Kumar, ${ }^{47}$ Arun Kumar, ${ }^{47}$ S. Ahuja, ${ }^{47}$ A. Bhardwaj, ${ }^{47}$ B. C. Choudhary, ${ }^{47}$ S. Malhotra, ${ }^{47}$ M. Naimuddin, ${ }^{47}$ K. Ranjan,${ }^{47}$ V. Sharma, ${ }^{47}$ R. K. Shivpuri ${ }^{47}$

S. Banerjee, ${ }^{48}$ S. Bhattacharya,${ }^{48}$ S. Dutta, ${ }^{48}$ B. Gomber, ${ }^{48}$ Sa. Jain,${ }^{48}$ Sh. Jain,${ }^{48}$ R. Khurana, ${ }^{48}$ S. Sarkar,${ }^{48}$ M. Sharan, ${ }^{48}$ A. Abdulsalam, ${ }^{49}$ R. K. Choudhury, ${ }^{49}$ D. Dutta ${ }^{49}$ S. Kailas, ${ }^{49}$ V. Kumar, ${ }^{49}$ P. Mehta, ${ }^{49}$ A. K. Mohanty, ${ }^{49, \mathrm{f}}$ L. M. Pant ${ }^{49}$ P. Shukla, ${ }^{49}$ T. Aziz, ${ }^{50}$ S. Ganguly, ${ }^{50}$ M. Guchait,${ }^{50, \mathrm{u}}$ M. Maity, ${ }^{50, v}$ G. Majumder,${ }^{50}$ K. Mazumdar, ${ }^{50}$ G. B. Mohanty, ${ }^{50}$ B. Parida,${ }^{50}$ K. Sudhakar,${ }^{50}$ N. Wickramage, ${ }^{50}$ S. Banerjee,${ }^{51}$ S. Dugad,${ }^{51}$ H. Arfaei, ${ }^{52}$ H. Bakhshiansohi, ${ }^{52, w}$ S. M. Etesami, ${ }^{52, \mathrm{x}}$ A. Fahim, ${ }^{52, w}$ M. Hashemi, ${ }^{52}$ H. Hesari, ${ }^{52}$ A. Jafari, ${ }^{52, w}$ M. Khakzad, ${ }^{52}$ M. Mohammadi Najafabadi,${ }^{52}$ S. Paktinat Mehdiabadi, ${ }^{52}$ B. Safarzadeh, ${ }^{52, y}$ M. Zeinali, ${ }^{52, x}$ M. Abbrescia, ${ }^{53 a, 53 b}$ L. Barbone, ${ }^{53 a, 53 b}$ C. Calabria, ${ }^{53 a, 53 b, f}$ S. S. Chhibra, ${ }^{53 a, 53 b}$ A. Colaleo, ${ }^{53 a}$ D. Creanza, ${ }^{53 a, 53 c}$ N. De Filippis, ${ }^{53 a, 53 c, f}$ M. De Palma, ${ }^{53 a, 53 b}$ L. Fiore,${ }^{53 a}$ G. Iaselli, ${ }^{53 a, 53 c}$ L. Lusito, ${ }^{53 a, 53 b}$ G. Maggi, ${ }^{53 a, 53 c}$ M. Maggi, ${ }^{53 a}$ B. Marangelli, ${ }^{53 a, 53 b}$ S. My, ${ }^{53 a, 53 c}$ S. Nuzzo, ${ }^{53 a, 53 b}$ N. Pacifico, ${ }^{53 a, 53 b}$ A. Pompili, ${ }^{53 a, 53 b}$ G. Pugliese, ${ }^{53 a, 53 c}$ G. Selvaggi, ${ }^{53 a, 53 b}$ L. Silvestris, ${ }^{53 a}$ G. Singh, ${ }^{53 a, 53 b}$ R. Venditti, ${ }^{53 a}$ G. Zito, ${ }^{53 a}$ G. Abbiendi, ${ }^{54 a}$ A. C. Benvenuti, ${ }^{54 a}$ D. Bonacorsi, ${ }^{54 a, 54 b}$ S. Braibant-Giacomelli, ${ }^{54 a, 54 b}$ L. Brigliadori, ${ }^{54 a, 54 b}$ P. Capiluppi, ${ }^{54 a, 54 b}$ A. Castro, ${ }^{54 a, 54 b}$ F. R. Cavallo, ${ }^{54 a}$ M. Cuffiani, ${ }^{54 a, 54 b}$ G. M. Dallavalle, ${ }^{54 a}$ F. Fabbri, ${ }^{54 a}$ A. Fanfani,${ }^{54 a, 54 b}$ D. Fasanella, ${ }^{54 a, 54 b, f}$ P. Giacomelli, ${ }^{54 a}$ C. Grandi, ${ }^{54 a}$ L. Guiducci, ${ }^{54 a, 54 b}$ S. Marcellini, ${ }^{54 a}$ G. Masetti, ${ }^{54 a}$ M. Meneghelli, ${ }^{54 a, 54 b, f}$ A. Montanari, ${ }^{54 a}$ F. L. Navarria, ${ }^{54 a, 54 b}$ F. Odorici, ${ }^{54 a}$ A. Perrotta, ${ }^{54 a}$ F. Primavera,${ }^{54 a, 54 b}$ A. M. Rossi, ${ }^{54 a, 54 b}$ T. Rovelli, ${ }^{54 a, 54 b}$ G. P. Siroli, ${ }^{54 a, 54 b}$ R. Travaglini, ${ }^{54 a, 54 b}$ S. Albergo, ${ }^{55 a, 55 b}$ G. Cappello, ${ }^{55 a, 55 b}$ M. Chiorboli, ${ }^{55 a, 55 b}$ S. Costa, ${ }^{55 a, 55 b}$ R. Potenza, ${ }^{55 a, 55 b}$ A. Tricomi ${ }^{55 a, 55 b}$ C. Tuve, ${ }^{55 a, 55 b}$ G. Barbagli, ${ }^{56 a}$ V. Ciulli, ${ }^{56 a, 56 b}$ C. Civinini, ${ }^{56 a}$ R. D'Alessandro, ${ }^{56 a, 56 b}$ E. Focardi, ${ }^{56 a, 56 b}$ S. Frosali, ${ }^{56 a, 56 b}$ E. Gallo, ${ }^{56 a}$ S. Gonzi, ${ }^{56 a, 56 b}$ M. Meschini, ${ }^{56 a}$ S. Paoletti, ${ }^{56 a}$ G. Sguazzoni, ${ }^{56 a}$ A. Tropiano, ${ }^{56 a}$ L. Benussi,${ }^{57}$ S. Bianco, ${ }^{57}$ S. Colafranceschi,${ }^{57, z}$ F. Fabbri, ${ }^{57}$ D. Piccolo,${ }^{57}$ P. Fabbricatore, ${ }^{58 a}$ R. Musenich, ${ }^{58 a}$ S. Tosi, ${ }^{58 a, 58 b}$ A. Benaglia, ${ }^{59 a, 59 b}$ F. De Guio, ${ }^{59 a, 59 b}$ L. Di Matteo, ${ }^{59 a, 59 b, f}$ S. Fiorendi, ${ }^{59,59 b}$ S. Gennai, ${ }^{59 a, f}$ A. Ghezzi, ${ }^{59 a, 59 b}$ S. Malvezzi, ${ }^{59 a}$ R. A. Manzoni, ${ }^{59 a, 59 b}$ A. Martelli, ${ }^{59 a, 59 b}$ A. Massironi, ${ }^{59 a, 59 b, f}$ D. Menasce, ${ }^{59 a}$ L. Moroni, ${ }^{59 a}$ M. Paganoni, ${ }^{59 a, 59 b}$ D. Pedrini, ${ }^{59 a}$ S. Ragazzi, ${ }^{59 a, 59 b}$ N. Redaelli, ${ }^{59 a}$ S. Sala, ${ }^{59 a}$ T. Tabarelli de Fatis, ${ }^{59 a, 59 b}$ S. Buontempo, ${ }^{60 a}$ C. A. Carrillo Montoya, ${ }^{60 a}$ N. Cavallo, ${ }^{60 a, a a}$

A. De Cosa,${ }^{60 a, 60 b, f}$ O. Dogangun ${ }^{60 a, 60 b}$ F. Fabozzi, ${ }^{60 a, a a}$ A. O. M. Iorio, ${ }^{60 a}$ L. Lista, ${ }^{60 a}$ S. Meola,${ }^{60 a, b b}$ M. Merola ${ }^{60 a, 60 b}$ P. Paolucci, ${ }^{60 a, f}$ P. Azzi ${ }^{61 \mathrm{a}}$ N. Bacchetta, ${ }^{61 \mathrm{a}, \mathrm{f}}$ D. Bisello, ${ }^{61 \mathrm{a}, 61 \mathrm{~b}}$ A. Branca, ${ }^{61 \mathrm{a}, 61 \mathrm{~b}, \mathrm{f}}$ R. Carlin, ${ }^{61 \mathrm{a}, 61 \mathrm{~b}}$ P. Checchia, ${ }^{61 a}$ T. Dorigo, ${ }^{61 a}$ U. Dosselli, ${ }^{61 \mathrm{a}}$ F. Gasparini, ${ }^{61 \mathrm{a}, 61 \mathrm{~b}}$ U. Gasparini, ${ }^{61 \mathrm{a}, 61 \mathrm{~b}}$ A. Gozzelino, ${ }^{61 \mathrm{a}}$

K. Kanishchev, ${ }^{61 \mathrm{a}, 61 \mathrm{c}}$ S. Lacaprara, ${ }^{61 \mathrm{a}}$ I. Lazzizzera, ${ }^{61 \mathrm{a}, 61 \mathrm{c}}$ M. Margoni, ${ }^{61 \mathrm{a}, 61 \mathrm{~b}}$ A. T. Meneguzzo, ${ }^{61 \mathrm{a}, 61 \mathrm{~b}}$ J. Pazzini, ${ }^{61 \mathrm{a}, 61 \mathrm{~b}}$ N. Pozzobon, ${ }^{61 \mathrm{a}, 61 \mathrm{~b}}$ P. Ronchese,${ }^{61 \mathrm{a}, 61 \mathrm{~b}}$ F. Simonetto, ${ }^{61 \mathrm{a}, 61 \mathrm{~b}}$ E. Torassa, ${ }^{61 \mathrm{a}}$ M. Tosi, ${ }^{61 \mathrm{a}, 61 \mathrm{~b}, \mathrm{f}}$

S. Vanini, ${ }^{61 a, 61 b}$ P. Zotto, ${ }^{61 a, 61 b}$ G. Zumerle, ${ }^{61 a, 61 b}$ M. Gabusi, ${ }^{62 a, 62 b}$ S. P. Ratti, ${ }^{62 a, 62 b}$ C. Riccardi, ${ }^{62 a, 62 b}$ P. Torre, ${ }^{62 a, 62 b}$ P. Vitulo, ${ }^{62 a, 62 b}$ M. Biasini, ${ }^{63 a, 63 b}$ G. M. Bilei, ${ }^{63 a}$ L. Fanò,${ }^{63 a, 63 b}$ P. Lariccia, ${ }^{63 a, 63 b}$ A. Lucaroni, ${ }^{63 a, 63 b, f}$ G. Mantovani, ${ }^{63 a, 63 b}$ M. Menichelli, ${ }^{63 a}$ A. Nappi,${ }^{63 a, 63 b, a}$ F. Romeo, ${ }^{63 a, 63 b}$ A. Saha, ${ }^{63 a}$ A. Santocchia, ${ }^{63 a, 63 b}$ A. Spiezia, ${ }^{63 a, 63 b}$ S. Taroni, ${ }^{63 a, 63 b}$ P. Azzurri, ${ }^{64 a, 64 c}$ G. Bagliesi, ${ }^{64 a}$ T. Boccali, ${ }^{64 a}$ G. Broccolo, ${ }^{64 a, 64 c}$ R. Castaldi, ${ }^{64 a}$ R. T. D’Agnolo, ${ }^{64 a, 64 c}$ R. Dell'Orso, ${ }^{64 a}$ F. Fiori, ${ }^{64 a, 64 b, f}$ L. Foà ${ }^{64 a, 64 c}$ A. Giassi, ${ }^{64 a}$ A. Kraan, ${ }^{64 a}$ F. Ligabue, ${ }^{64 a, 64 c}$ T. Lomtadze, ${ }^{64 a}$ L. Martini, ${ }^{64 a, c c}$ A. Messineo, ${ }^{64 a, 64 b}$ F. Palla, ${ }^{64 a}$ A. Rizzi, ${ }^{64 a, 64 b}$ A. T. Serban, ${ }^{64 a, d d}$ P. Spagnolo, ${ }^{64 a}$ P. Squillacioti, ${ }^{64 a, f}$ R. Tenchini ${ }^{64 a}$ G. Tonelli ${ }^{64 a, 64 b, f}$ A. Venturi, ${ }^{64 a}$ P. G. Verdini, ${ }^{64 a}$ L. Barone,${ }^{65 a, 65 b}$ F. Cavallari, ${ }^{65 a}$ D. Del Re,${ }^{65 a, 65 b}$ M. Diemoz, ${ }^{65 a}$ C. Fanelli, ${ }^{65 a}$ M. Grassi,${ }^{65 a, 65 b, f}$ E. Longo, ${ }^{65 a, 65 b}$ P. Meridiani, ${ }^{65 a, f}$ F. Micheli, ${ }^{65 a, 65 b}$ S. Nourbakhsh, ${ }^{65 a, 65 b}$ G. Organtini, ${ }^{65 a, 65 b}$ R. Paramatti, ${ }^{65 a}$ S. Rahatlou, ${ }^{65 a, 65 b}$ M. Sigamani, ${ }^{65 a}$ L. Soffi, ${ }^{65 a, 65 b}$ N. Amapane, ${ }^{66 a, 66 b}$ R. Arcidiacono, ${ }^{66 a, 66 \mathrm{c}}$ S. Argiro, ${ }^{66 \mathrm{a}, 66 \mathrm{~b}}$ M. Arneodo, ${ }^{66 \mathrm{a}, 66 \mathrm{c}}$ C. Biino, ${ }^{66 \mathrm{a}} \mathrm{N}$. Cartiglia, ${ }^{66 \mathrm{a}}$ M. Costa, ${ }^{66 a, 66 b}$ P. De Remigis, ${ }^{66 a}$ N. Demaria, ${ }^{66 a}$ C. Mariotti, ${ }^{66 a, f}$ S. Maselli, ${ }^{66 a}$ E. Migliore, ${ }^{66 a, 66 b}$ V. Monaco, ${ }^{66 a, 66 b}$ M. Musich, ${ }^{66 a, f}$ M. M. Obertino, ${ }^{66 a, 66 c}$ N. Pastrone, ${ }^{66 a}$ M. Pelliccioni, ${ }^{66 a}$ A. Potenza, ${ }^{66 a, 66 b}$ A. Romero, ${ }^{66 a, 66 b}$ R. Sacchi, ${ }^{66 a, 66 b}$ A. Solano, ${ }^{66 a, 66 b}$ A. Staiano, ${ }^{66 a}$ A. Vilela Pereira, ${ }^{66 a}$ S. Belforte ${ }^{67 a}$ V. Candelise, ${ }^{67 a, 67 b}$ F. Cossutti ${ }^{67 a}$ G. Della Ricca ${ }^{67 a, 67 b}$ B. Gobbo, ${ }^{67 a}$ M. Marone,${ }^{67 a, 67 b, f}$ D. Montanino, ${ }^{67 a, 67 b, f}$ A. Penzo, ${ }^{67 a}$ A. Schizzi, ${ }^{67 a, 67 b}$ S. G. Heo, ${ }^{53}$ T. Y. Kim, ${ }^{53}$ S. K. Nam ${ }^{53}$ S. Chang, ${ }^{54}$ D. H. Kim,${ }^{54}$ G. N. Kim, ${ }^{54}$ D. J. Kong, ${ }^{54}$ H. Park, ${ }^{54}$ S. R. Ro, ${ }^{54}$ D. C. Son,${ }^{54}$ T. Son,${ }^{54}$ J. Y. Kim, ${ }^{55}$ Zero J. Kim, ${ }^{55}$ S. Song, ${ }^{55}$ S. Choi, ${ }^{56}$ D. Gyun, ${ }^{56}$ B. Hong,${ }^{56}$ M. Jo, ${ }^{56}$ H. Kim, ${ }^{56}$ T. J. Kim, ${ }^{56}$ K. S. Lee, ${ }^{56}$ D. H. Moon, ${ }^{56}$ S. K. Park, ${ }^{56}$ M. Choi,${ }^{57}$ J. H. Kim, ${ }^{57}$ C. Park, ${ }^{57}$ 
I. C. Park,${ }^{57}$ S. Park,${ }^{57}$ G. Ryu,${ }^{57}$ Y. Cho,${ }^{58}$ Y. Choi, ${ }^{58}$ Y. K. Choi,${ }^{58}$ J. Goh ${ }^{58}$ M. S. Kim, ${ }^{58}$ E. Kwon, ${ }^{58}$ B. Lee,${ }^{58}$ J. Lee, ${ }^{58}$ S. Lee, ${ }^{58}$ H. Seo,${ }^{58}$ I. Yu, ${ }^{58}$ M. J. Bilinskas, ${ }^{59}$ I. Grigelionis,${ }^{59}$ M. Janulis, ${ }^{59}$ A. Juodagalvis, ${ }^{59}$

H. Castilla-Valdez, ${ }^{60}$ E. De La Cruz-Burelo, ${ }^{60}$ I. Heredia-de La Cruz,${ }^{60}$ R. Lopez-Fernandez, ${ }^{60}$ R. Magaña Villalba, ${ }^{60}$

J. Martínez-Ortega, ${ }^{60}$ A. Sánchez-Hernández ${ }^{60}$ L. M. Villasenor-Cendejas, ${ }^{60}$ S. Carrillo Moreno, ${ }^{61}$

F. Vazquez Valencia ${ }^{61}$ H. A. Salazar Ibarguen, ${ }^{62}$ E. Casimiro Linares,${ }^{63}$ A. Morelos Pineda,${ }^{63}$ M. A. Reyes-Santos, ${ }^{63}$

D. Krofcheck, ${ }^{64}$ A. J. Bell ${ }^{65}$ P. H. Butler, ${ }^{65}$ R. Doesburg, ${ }^{65}$ S. Reucroft,${ }^{65}$ H. Silverwood, ${ }^{65}$ M. Ahmad, ${ }^{66}$

M. H. Ansari, ${ }^{66}$ M. I. Asghar,${ }^{66}$ H. R. Hoorani, ${ }^{66}$ S. Khalid,${ }^{66}$ W. A. Khan, ${ }^{66}$ T. Khurshid, ${ }^{66}$ S. Qazi, ${ }^{66}$ M. A. Shah, ${ }^{66}$ M. Shoaib, ${ }^{66}$ H. Bialkowska, ${ }^{67}$ B. Boimska,${ }^{67}$ T. Frueboes, ${ }^{67}$ R. Gokieli, ${ }^{67}$ M. Górski, ${ }^{67}$ M. Kazana, ${ }^{67}$ K. Nawrocki, ${ }^{67}$ K. Romanowska-Rybinska, ${ }^{67}$ M. Szleper, ${ }^{67}$ G. Wrochna,${ }^{67}$ P. Zalewski, ${ }^{67}$ G. Brona,${ }^{68}$ K. Bunkowski, ${ }^{68}$ M. Cwiok, ${ }^{68}$ W. Dominik, ${ }^{68}$ K. Doroba, ${ }^{68}$ A. Kalinowski, ${ }^{68}$ M. Konecki, ${ }^{68}$ J. Krolikowski, ${ }^{68}$ N. Almeida ${ }^{69}$ P. Bargassa, ${ }^{69}$

A. David ${ }^{69}$ P. Faccioli, ${ }^{69}$ P. G. Ferreira Parracho, ${ }^{69}$ M. Gallinaro, ${ }^{69}$ J. Seixas, ${ }^{69}$ J. Varela, ${ }^{69}$ P. Vischia, ${ }^{69}$

I. Belotelov, ${ }^{70}$ P. Bunin,${ }^{70}$ M. Gavrilenko,${ }^{70}$ I. Golutvin, ${ }^{70}$ A. Kamenev,${ }^{70}$ V. Karjavin, ${ }^{70}$ G. Kozlov,${ }^{70}$ A. Lanev, ${ }^{70}$ A. Malakhov, ${ }^{70}$ P. Moisenz ${ }^{70}$ V. Palichik, ${ }^{70}$ V. Perelygin, ${ }^{70}$ M. Savina,${ }^{70}$ S. Shmatov, ${ }^{70}$ V. Smirnov, ${ }^{70}$ A. Volodko,${ }^{70}$ A. Zarubin, ${ }^{70}$ S. Evstyukhin, ${ }^{71}$ V. Golovtsov, ${ }^{71}$ Y. Ivanov, ${ }^{71}$ V. Kim, ${ }^{71}$ P. Levchenko, ${ }^{71}$ V. Murzin, ${ }^{71}$ V. Oreshkin, ${ }^{71}$

I. Smirnov, ${ }^{71}$ V. Sulimov, ${ }^{71}$ L. Uvarov, ${ }^{71}$ S. Vavilov, ${ }^{71}$ A. Vorobyev, ${ }^{71}$ An. Vorobyev, ${ }^{71}$ Yu. Andreev, ${ }^{72}$

A. Dermenev, ${ }^{72}$ S. Gninenko, ${ }^{72}$ N. Golubev, ${ }^{72}$ M. Kirsanov, ${ }^{72}$ N. Krasnikov, ${ }^{72}$ V. Matveev, ${ }^{72}$ A. Pashenkov, ${ }^{72}$

D. Tlisov, ${ }^{72}$ A. Toropin, ${ }^{72}$ V. Epshteyn, ${ }^{73}$ M. Erofeeva, ${ }^{73}$ V. Gavrilov,${ }^{73}$ M. Kossov, ${ }^{73}$ N. Lychkovskaya, ${ }^{73}$ V. Popov, ${ }^{73}$

G. Safronov, ${ }^{73}$ S. Semenov, ${ }^{73}$ V. Stolin, ${ }^{73}$ E. Vlasov, ${ }^{73}$ A. Zhokin,,${ }^{73}$ A. Belyaev, ${ }^{74}$ E. Boos,${ }^{74}$ V. Bunichev, ${ }^{74}$

M. Dubinin, ${ }^{74, \mathrm{e}}$ L. Dudko, ${ }^{74}$ A. Gribushin, ${ }^{74}$ V. Klyukhin, ${ }^{74}$ O. Kodolova, ${ }^{74}$ I. Lokhtin, ${ }^{74}$ A. Markina, ${ }^{74}$

S. Obraztsov, ${ }^{74}$ M. Perfilov,${ }^{74}$ S. Petrushanko, ${ }^{74}$ A. Popov, ${ }^{74}$ L. Sarycheva, ${ }^{74, a}$ V. Savrin, ${ }^{74}$ A. Snigirev,${ }^{74}$

V. Andreev,${ }^{75}$ M. Azarkin, ${ }^{75}$ I. Dremin, ${ }^{75}$ M. Kirakosyan,${ }^{75}$ A. Leonidov,${ }^{75}$ G. Mesyats, ${ }^{75}$ S. V. Rusakov, ${ }^{75}$

A. Vinogradov, ${ }^{75}$ I. Azhgirey, ${ }^{76}$ I. Bayshev,${ }^{76}$ S. Bitioukov, ${ }^{76}$ V. Grishin, ${ }^{76, f}$ V. Kachanov, ${ }^{76}$ D. Konstantinov, ${ }^{76}$

A. Korablev, ${ }^{76}$ V. Krychkine, ${ }^{76}$ V. Petrov, ${ }^{76}$ R. Ryutin, ${ }^{76}$ A. Sobol,${ }^{76}$ L. Tourtchanovitch, ${ }^{76}$ S. Troshin, ${ }^{76}$ N. Tyurin, ${ }^{76}$

A. Uzunian, ${ }^{76}$ A. Volkov, ${ }^{76}$ P. Adzic, ${ }^{77, \text { ee }}$ M. Djordjevic, ${ }^{77}$ M. Ekmedzic, ${ }^{77}$ D. Krpic, ${ }^{77, \text { ee }}$ J. Milosevic, ${ }^{77}$

M. Aguilar-Benitez,${ }^{78}$ J. Alcaraz Maestre ${ }^{78}$ P. Arce,${ }^{78}$ C. Battilana, ${ }^{78}$ E. Calvo,${ }^{78}$ M. Cerrada,${ }^{78}$

M. Chamizo Llatas, ${ }^{78}$ N. Colino, ${ }^{78}$ B. De La Cruz ${ }^{78}$ A. Delgado Peris, ${ }^{78}$ D. Domínguez Vázquez, ${ }^{78}$

C. Fernandez Bedoya,${ }^{78}$ J. P. Fernández Ramos, ${ }^{78}$ A. Ferrando, ${ }^{78}$ J. Flix,${ }^{78}$ M. C. Fouz, ${ }^{78}$ P. Garcia-Abia,${ }^{78}$

O. Gonzalez Lopez, ${ }^{78}$ S. Goy Lopez,${ }^{78}$ J. M. Hernandez, ${ }^{78}$ M. I. Josa, ${ }^{78}$ G. Merino, ${ }^{78}$ J. Puerta Pelayo, ${ }^{78}$

A. Quintario Olmeda, ${ }^{78}$ I. Redondo,${ }^{78}$ L. Romero, ${ }^{78}$ J. Santaolalla ${ }^{78}$ M. S. Soares,${ }^{78}$ C. Willmott, ${ }^{78}$ C. Albajar, ${ }^{79}$

G. Codispoti, ${ }^{79}$ J.F. de Trocóniz, ${ }^{79}$ H. Brun,${ }^{80}$ J. Cuevas, ${ }^{80}$ J. Fernandez Menendez, ${ }^{80}$ S. Folgueras,${ }^{80}$

I. Gonzalez Caballero, ${ }^{80}$ L. Lloret Iglesias, ${ }^{80}$ J. Piedra Gomez, ${ }^{80}$ J. A. Brochero Cifuentes, ${ }^{81}$ I. J. Cabrillo, ${ }^{81}$

A. Calderon, ${ }^{81}$ S. H. Chuang, ${ }^{81}$ J. Duarte Campderros, ${ }^{81}$ M. Felcini, ${ }^{81, f f}$ M. Fernandez, ${ }^{81}$ G. Gomez, ${ }^{81}$

J. Gonzalez Sanchez, ${ }^{81}$ A. Graziano, ${ }^{81}$ C. Jorda,${ }^{81}$ A. Lopez Virto, ${ }^{81}$ J. Marco,${ }^{81}$ R. Marco, ${ }^{81}$ C. Martinez Rivero, ${ }^{81}$ F. Matorras, ${ }^{81}$ F. J. Munoz Sanchez, ${ }^{81}$ T. Rodrigo, ${ }^{81}$ A. Y. Rodríguez-Marrero, ${ }^{81}$ A. Ruiz-Jimeno, ${ }^{81}$ L. Scodellaro, ${ }^{81}$ M. Sobron Sanudo, ${ }^{81}$ I. Vila, ${ }^{81}$ R. Vilar Cortabitarte, ${ }^{81}$ D. Abbaneo, ${ }^{82}$ E. Auffray, ${ }^{82}$ G. Auzinger, ${ }^{82}$ M. Bachtis, ${ }^{82}$ P. Baillon, ${ }^{82}$ A. H. Ball, ${ }^{82}$ D. Barney, ${ }^{82}$ J. F. Benitez,${ }^{82}$ C. Bernet, ${ }^{82, g}$ G. Bianchi, ${ }^{82}$ P. Bloch,${ }^{82}$ A. Bocci, ${ }^{82}$

A. Bonato, ${ }^{82}$ C. Botta, ${ }^{82}$ H. Breuker, ${ }^{82}$ T. Camporesi, ${ }^{82}$ G. Cerminara, ${ }^{82}$ T. Christiansen, ${ }^{82}$ J. A. Coarasa Perez, ${ }^{82}$

D. D'Enterria, ${ }^{82}$ A. Dabrowski, ${ }^{82}$ A. De Roeck, ${ }^{82}$ S. Di Guida, ${ }^{82}$ M. Dobson,${ }^{82}$ N. Dupont-Sagorin,${ }^{82}$

A. Elliott-Peisert, ${ }^{82}$ B. Frisch, ${ }^{82}$ W. Funk,${ }^{82}$ G. Georgiou, ${ }^{82}$ M. Giffels, ${ }^{82}$ D. Gigi, ${ }^{82}$ K. Gill, ${ }^{82}$ D. Giordano, ${ }^{82}$

M. Giunta, ${ }^{82}$ F. Glege, ${ }^{82}$ R. Gomez-Reino Garrido, ${ }^{82}$ P. Govoni, ${ }^{82}$ S. Gowdy, ${ }^{82}$ R. Guida, ${ }^{82}$ M. Hansen, ${ }^{82}$ P. Harris, ${ }^{82}$

C. Hartl, ${ }^{82}$ J. Harvey, ${ }^{82}$ B. Hegner, ${ }^{82}$ A. Hinzmann, ${ }^{82}$ V. Innocente, ${ }^{82}$ P. Janot, ${ }^{82}$ K. Kaadze,${ }^{82}$ E. Karavakis ${ }^{82}$

K. Kousouris, ${ }^{82}$ P. Lecoq ${ }^{82}$ Y.-J. Lee,${ }^{82}$ P. Lenzi, ${ }^{82}$ C. Lourenço,${ }^{82}$ N. Magini, ${ }^{82}$ T. Mäki, ${ }^{82}$ M. Malberti, ${ }^{82}$

L. Malgeri, ${ }^{82}$ M. Mannelli, ${ }^{82}$ L. Masetti, ${ }^{82}$ F. Meijers, ${ }^{82}$ S. Mersi, ${ }^{82}$ E. Meschi, ${ }^{82}$ R. Moser, ${ }^{82}$ M. U. Mozer, ${ }^{82}$

M. Mulders, ${ }^{82}$ P. Musella, ${ }^{82}$ E. Nesvold, ${ }^{82}$ T. Orimoto, ${ }^{82}$ L. Orsini, ${ }^{82}$ E. Palencia Cortezon, ${ }^{82}$ E. Perez, ${ }^{82}$

L. Perrozzi, ${ }^{82}$ A. Petrilli, ${ }^{82}$ A. Pfeiffer, ${ }^{82}$ M. Pierini, ${ }^{82}$ M. Pimiä, ${ }^{82}$ D. Piparo, ${ }^{82}$ G. Polese, ${ }^{82}$ L. Quertenmont, ${ }^{82}$

A. Racz, ${ }^{82}$ W. Reece, ${ }^{82}$ J. Rodrigues Antunes, ${ }^{82}$ G. Rolandi, ${ }^{82, g g}$ C. Rovelli, ${ }^{82, \text { hh }}$ M. Rovere, ${ }^{82}$ H. Sakulin, ${ }^{82}$

F. Santanastasio, ${ }^{82}$ C. Schäfer, ${ }^{82}$ C. Schwick, ${ }^{82}$ I. Segoni, ${ }^{82}$ S. Sekmen, ${ }^{82}$ A. Sharma, ${ }^{82}$ P. Siegrist, ${ }^{82}$ P. Silva, ${ }^{82}$

M. Simon, ${ }^{82}$ P. Sphicas, ${ }^{82, i i}$ D. Spiga, ${ }^{82}$ A. Tsirou, ${ }^{82}$ G. I. Veres, ${ }^{82, t}$ J. R. Vlimant, ${ }^{82}$ H. K. Wöhri, ${ }^{82}$ S. D. Worm, ${ }^{82, j j}$

W. D. Zeuner, ${ }^{82}$ W. Bertl, ${ }^{83}$ K. Deiters, ${ }^{83}$ W. Erdmann, ${ }^{83}$ K. Gabathuler, ${ }^{83}$ R. Horisberger, ${ }^{83}$ Q. Ingram,${ }^{83}$

H. C. Kaestli, ${ }^{83}$ S. König, ${ }^{83}$ D. Kotlinski, ${ }^{83}$ U. Langenegger, ${ }^{83}$ F. Meier, ${ }^{83}$ D. Renker, ${ }^{83}$ T. Rohe, ${ }^{83}$ J. Sibille, ${ }^{83, k k}$ L. Bäni, ${ }^{84}$ P. Bortignon, ${ }^{84}$ M. A. Buchmann, ${ }^{84}$ B. Casal,${ }^{84}$ N. Chanon, ${ }^{84}$ A. Deisher, ${ }^{84}$ G. Dissertori, ${ }^{84}$ M. Dittmar,${ }^{84}$ 
M. Donegà, ${ }^{84}$ M. Dünser ${ }^{84}$ J. Eugster,${ }^{84}$ K. Freudenreich,${ }^{84}$ C. Grab,${ }^{84}$ D. Hits,${ }^{84}$ P. Lecomte,${ }^{84}$ W. Lustermann, ${ }^{84}$ A. C. Marini, ${ }^{84}$ P. Martinez Ruiz del Arbol, ${ }^{84}$ N. Mohr, ${ }^{84}$ F. Moortgat ${ }^{84}$ C. Nägeli, ${ }^{84,11}$ P. Nef,${ }^{84}$ F. Nessi-Tedaldi, ${ }^{84}$ F. Pandolfi, ${ }^{84}$ L. Pape,${ }^{84}$ F. Pauss,${ }^{84}$ M. Peruzzi ${ }^{84}$ F. J. Ronga,${ }^{84}$ M. Rossini,${ }^{84}$ L. Sala, ${ }^{84}$ A. K. Sanchez ${ }^{84}$ A. Starodumov ${ }^{84, \mathrm{~mm}}$ B. Stieger, ${ }^{84}$ M. Takahashi, ${ }^{84}$ L. Tauscher, ${ }^{84, a}$ A. Thea, ${ }^{84}$ K. Theofilatos, ${ }^{84}$ D. Treille,${ }^{84}$ C. Urscheler, ${ }^{84}$ R. Wallny, ${ }^{84}$ H. A. Weber ${ }^{84}$ L. Wehrli, ${ }^{84}$ C. Amsler,${ }^{85}$ V. Chiochia, ${ }^{85}$ S. De Visscher,${ }^{85}$ C. Favaro, ${ }^{85}$ M. Ivova Rikova, ${ }^{85}$ B. Millan Mejias, ${ }^{85}$ P. Otiougova, ${ }^{85}$ P. Robmann, ${ }^{85}$ H. Snoek,${ }^{85}$ S. Tupputi, ${ }^{85}$ M. Verzetti, ${ }^{85}$ Y. H. Chang, ${ }^{86}$ K. H. Chen, ${ }^{86}$ C. M. Kuo, ${ }^{86}$ S. W. Li ${ }^{86}$ W. Lin, ${ }^{86}$ Z. K. Liu, ${ }^{86}$ Y. J. Lu, ${ }^{86}$ D. Mekterovic, ${ }^{86}$ A. P. Singh, ${ }^{86}$ R. Volpe, ${ }^{86}$ S. S. Yu, ${ }^{86}$ P. Bartalini, ${ }^{87}$ P. Chang, ${ }^{87}$ Y. H. Chang, ${ }^{87}$ Y. W. Chang, ${ }^{87}$ Y. Chao,${ }^{87}$ K. F. Chen, ${ }^{87}$ C. Dietz, ${ }^{87}$ U. Grundler, ${ }^{87}$ W.-S. Hou ${ }^{87}$ Y. Hsiung, ${ }^{87}$ K. Y. Kao, ${ }^{87}$ Y. J. Lei, ${ }^{87}$ R.-S. Lu, ${ }^{87}$ D. Majumder, ${ }^{87}$ E. Petrakou, ${ }^{87}$ X. Shi,${ }^{87}$ J. G. Shiu, ${ }^{87}$ Y. M. Tzeng, ${ }^{87}$ X. Wan, ${ }^{87}$ M. Wang, ${ }^{87}$ A. Adiguzel,,${ }^{88}$ M. N. Bakirci, ${ }^{88, \text { nn }}$ S. Cerci ${ }^{88, \text { oo }}$ C. Dozen, ${ }^{88}$ I. Dumanoglu ${ }^{88}$ E. Eskut, ${ }^{88}$ S. Girgis,${ }^{88}$ G. Gokbulut ${ }^{88}$ E. Gurpinar, ${ }^{88}$ I. Hos, ${ }^{88}$ E. E. Kangal, ${ }^{88}$ T. Karaman ${ }^{88}$ G. Karapinar, ${ }^{88, p p}$ A. Kayis Topaksu, ${ }^{88}$ G. Onengut, ${ }^{88}$ K. Ozdemir, ${ }^{88}$ S. Ozturk, ${ }^{88, q q}$ A. Polatoz, ${ }^{88}$ K. Sogut, ${ }^{88, \text { rr }}$ D. Sunar Cerci,${ }^{88, \text { oo }}$ B. Tali, ${ }^{88, o o}$ H. Topakli, ${ }^{88, \text {,n }}$ L. N. Vergili, ${ }^{88}$ M. Vergili, ${ }^{88}$ I. V. Akin, ${ }^{89}$ T. Aliev, ${ }^{89}$ B. Bilin, ${ }^{89}$ S. Bilmis, ${ }^{89}$ M. Deniz,${ }^{89}$ H. Gamsizkan, ${ }^{89}$ A. M. Guler, ${ }^{89}$ K. Ocalan, ${ }^{89}$ A. Ozpineci, ${ }^{89}$ M. Serin, ${ }^{89}$ R. Sever, ${ }^{89}$ U.E. Surat,${ }^{89}$ M. Yalvac, ${ }^{89}$ E. Yildirim, ${ }^{89}$ M. Zeyrek,${ }^{89}$ E. Gülmez, ${ }^{90}$ B. Isildak, ${ }^{90, \text { ss }}$ M. Kaya, ${ }^{90, t t}$ O. Kaya,${ }^{90, t t}$ S. Ozkorucuklu, ${ }^{90, \text { uu }}$ N. Sonmez, ${ }^{90, v v}$ K. Cankocak, ${ }^{91}$ L. Levchuk, ${ }^{92}$ F. Bostock, ${ }^{93}$ J. J. Brooke, ${ }^{93}$ E. Clement,,${ }^{93}$ D. Cussans, ${ }^{93}$ H. Flacher, ${ }^{93}$ R. Frazier, ${ }^{93}$ J. Goldstein,${ }^{93}$ M. Grimes, ${ }^{93}$ G. P. Heath, ${ }^{93}$ H. F. Heath, ${ }^{93}$ L. Kreczko, ${ }^{93}$ S. Metson,${ }^{93}$ D. M. Newbold, ${ }^{93, j j}$ K. Nirunpong, ${ }^{93}$ A. Poll, ${ }^{93}$ S. Senkin, ${ }^{93}$ V. J. Smith,${ }^{93}$ T. Williams,${ }^{93}$ L. Basso, ${ }^{94, w w}$ K. W. Bell, ${ }^{94}$ A. Belyaev, ${ }^{94, w w}$ C. Brew,${ }^{94}$ R. M. Brown, ${ }^{94}$ D. J. A. Cockerill, ${ }^{94}$ J. A. Coughlan, ${ }^{94}$ K. Harder, ${ }^{94}$ S. Harper,,${ }^{94}$ J. Jackson, ${ }^{94}$ B. W. Kennedy, ${ }^{94}$ E. Olaiya,${ }^{94}$ D. Petyt,${ }^{94}$ B. C. Radburn-Smith, ${ }^{94}$ C. H. Shepherd-Themistocleous, ${ }^{94}$ I. R. Tomalin, ${ }^{94}$ W. J. Womersley, ${ }^{94}$ R. Bainbridge, ${ }^{95}$ G. Ball, ${ }^{95}$ R. Beuselinck, ${ }^{95}$ O. Buchmuller,${ }^{95}$ D. Colling, ${ }^{95}$ N. Cripps, ${ }^{95}$ M. Cutajar,${ }^{95}$ P. Dauncey,${ }^{95}$ G. Davies, ${ }^{95}$ M. Della Negra, ${ }^{95}$ W. Ferguson, ${ }^{95}$ J. Fulcher, ${ }^{95}$ D. Futyan, ${ }^{95}$ A. Gilbert, ${ }^{95}$ A. Guneratne Bryer ${ }^{95}$ G. Hall, ${ }^{95}$ Z. Hatherell,${ }^{95}$ J. Hays,${ }^{95}$ G. Iles, ${ }^{95}$ M. Jarvis,,${ }^{95}$ G. Karapostoli, ${ }^{95}$ L. Lyons, ${ }^{95}$ A.-M. Magnan,,${ }^{95}$ J. Marrouche, ${ }^{95}$ B. Mathias,${ }^{95}$ R. Nandi, ${ }^{95}$ J. Nash,${ }^{95}$ A. Nikitenko, ${ }^{95, m m}$ A. Papageorgiou, ${ }^{95}$ J. Pela, ${ }^{95}$ M. Pesaresi,${ }^{95}$ K. Petridis, ${ }^{95}$ M. Pioppi ${ }^{95, x x}$ D. M. Raymond, ${ }^{95}$ S. Rogerson, ${ }^{95}$ A. Rose, ${ }^{95}$ M. J. Ryan, ${ }^{95}$ C. Seez, ${ }^{95}$ P. Sharp, ${ }^{95, a}$ A. Sparrow, ${ }^{95}$ M. Stoye,${ }^{95}$ A. Tapper, ${ }^{95}$ M. Vazquez Acosta,${ }^{95}$ T. Virdee, ${ }^{95}$ S. Wakefield,${ }^{95}$ N. Wardle,,${ }^{95}$ T. Whyntie,${ }^{95}$ M. Chadwick, ${ }^{96}$ J. E. Cole, ${ }^{96}$ P. R. Hobson, ${ }^{96}$ A. Khan, ${ }^{96}$ P. Kyberd,${ }^{96}$ D. Leggat,${ }^{96}$ D. Leslie, ${ }^{96}$ W. Martin, ${ }^{96}$ I. D. Reid,${ }^{96}$ P. Symonds, ${ }^{96}$ L. Teodorescu, ${ }^{96}$ M. Turner,${ }^{96}$ K. Hatakeyama, ${ }^{97}$ H. Liu, ${ }^{97}$ T. Scarborough,${ }^{97}$ O. Charaf,${ }^{98}$ C. Henderson, ${ }^{98}$ P. Rumerio, ${ }^{98}$ A. Avetisyan, ${ }^{99}$ T. Bose, ${ }^{99}$ C. Fantasia, ${ }^{99}$ A. Heister, ${ }^{99}$ J. St. John, ${ }^{99}$ P. Lawson, ${ }^{99}$ D. Lazic, ${ }^{99}$ J. Rohlf, ${ }^{99}$ D. Sperka, ${ }^{99}$ L. Sulak, ${ }^{99}$ J. Alimena, ${ }^{100}$ S. Bhattacharya, ${ }^{100}$ D. Cutts, ${ }^{100}$ A. Ferapontov, ${ }^{100}$ U. Heintz, ${ }^{100}$ S. Jabeen, ${ }^{100}$ G. Kukartsev, ${ }^{100}$ E. Laird, ${ }^{100}$ G. Landsberg, ${ }^{100}$ M. Luk, ${ }^{100}$ M. Narain, ${ }^{100}$ D. Nguyen, ${ }^{100}$ M. Segala, ${ }^{100}$ T. Sinthuprasith, ${ }^{100}$ T. Speer,${ }^{100}$ K. V. Tsang, ${ }^{100}$ R. Breedon, ${ }^{101}$ G. Breto,,${ }^{101}$

M. Calderon De La Barca Sanchez, ${ }^{101}$ S. Chauhan, ${ }^{101}$ M. Chertok, ${ }^{101}$ J. Conway, ${ }^{101}$ R. Conway, ${ }^{101}$ P. T. Cox, ${ }^{101}$ J. Dolen, ${ }^{101}$ R. Erbacher, ${ }^{101}$ M. Gardner, ${ }^{101}$ R. Houtz, ${ }^{101}$ W. Ko, ${ }^{101}$ A. Kopecky, ${ }^{101}$ R. Lander, ${ }^{101}$ T. Miceli, ${ }^{101}$ D. Pellett, ${ }^{101}$ F. Ricci-tam, ${ }^{101}$ B. Rutherford,${ }^{101}$ M. Searle, ${ }^{101}$ J. Smith, ${ }^{101}$ M. Squires, ${ }^{101}$ M. Tripathi,,${ }^{101}$

R. Vasquez Sierra, ${ }^{101}$ V. Andreev, ${ }^{102}$ D. Cline, ${ }^{102}$ R. Cousins, ${ }^{102}$ J. Duris,${ }^{102}$ S. Erhan,${ }^{102}$ P. Everaerts, ${ }^{102}$ C. Farrell, ${ }^{102}$ J. Hauser, ${ }^{102}$ M. Ignatenko, ${ }^{102}$ C. Jarvis, ${ }^{102}$ C. Plager, ${ }^{102}$ G. Rakness, ${ }^{102}$ P. Schlein, ${ }^{102, a}$ P. Traczyk,${ }^{102}$ V. Valuev, ${ }^{102}$ M. Weber, ${ }^{102}$ J. Babb, ${ }^{103}$ R. Clare, ${ }^{103}$ M. E. Dinardo, ${ }^{103}$ J. Ellison, ${ }^{103}$ J. W. Gary, ${ }^{103}$ F. Giordano, ${ }^{103}$

G. Hanson, ${ }^{103}$ G. Y. Jeng, ${ }^{103, y y}$ H. Liu, ${ }^{103}$ O. R. Long, ${ }^{103}$ A. Luthra, ${ }^{103}$ H. Nguyen, ${ }^{103}$ S. Paramesvaran, ${ }^{103}$

J. Sturdy, ${ }^{103}$ S. Sumowidagdo, ${ }^{103}$ R. Wilken, ${ }^{103}$ S. Wimpenny, ${ }^{103}$ W. Andrews, ${ }^{104}$ J. G. Branson, ${ }^{104}$ G. B. Cerati, ${ }^{104}$ S. Cittolin, ${ }^{104}$ D. Evans, ${ }^{104}$ F. Golf, ${ }^{104}$ A. Holzner, ${ }^{104}$ R. Kelley, ${ }^{104}$ M. Lebourgeois, ${ }^{104}$ J. Letts, ${ }^{104}$ I. Macneill, ${ }^{104}$ B. Mangano, ${ }^{104}$ S. Padhi, ${ }^{104}$ C. Palmer, ${ }^{104}$ G. Petrucciani,${ }^{104}$ M. Pieri, ${ }^{104}$ M. Sani, ${ }^{104}$ V. Sharma, ${ }^{104}$ S. Simon, ${ }^{104}$ E. Sudano, ${ }^{104}$ M. Tadel, ${ }^{104}$ Y. Tu, ${ }^{104}$ A. Vartak, ${ }^{104}$ S. Wasserbaech,,${ }^{104, z z}$ F. Würthwein, ${ }^{104}$ A. Yagil,,${ }^{104}$ J. Yoo, ${ }^{104}$ D. Barge, ${ }^{105}$ R. Bellan, ${ }^{105}$ C. Campagnari, ${ }^{105}$ M. D'Alfonso, ${ }^{105}$ T. Danielson, ${ }^{105}$ K. Flowers, ${ }^{105}$ P. Geffert, ${ }^{105}$ J. Incandela, ${ }^{105}$ C. Justus, ${ }^{105}$ P. Kalavase, ${ }^{105}$ S. A. Koay, ${ }^{105}$ D. Kovalskyi,,${ }^{105}$ V. Krutelyov, ${ }^{105}$ S. Lowette, ${ }^{105}$ N. Mccoll, ${ }^{105}$ V. Pavlunin, ${ }^{105}$ F. Rebassoo, ${ }^{105}$ J. Ribnik, ${ }^{105}$ J. Richman, ${ }^{105}$ R. Rossin, ${ }^{105}$ D. Stuart, ${ }^{105}$ W. To, ${ }^{105}$ C. West ${ }^{105}$ A. Apresyan, ${ }^{106}$ A. Bornheim, ${ }^{106}$ Y. Chen, ${ }^{106}$ E. Di Marco, ${ }^{106}$ J. Duarte, ${ }^{106}$ M. Gataullin,,${ }^{106}$ Y. Ma, ${ }^{106}$ A. Mott, ${ }^{106}$ H. B. Newman, ${ }^{106}$ C. Rogan, ${ }^{106}$ M. Spiropulu, ${ }^{106}$ V. Timciuc, ${ }^{106}$ J. Veverka, ${ }^{106}$ R. Wilkinson, ${ }^{106}$ S. Xie, ${ }^{106}$ Y. Yang, ${ }^{106}$ R. Y. Zhu, ${ }^{106}$ B. Akgun, ${ }^{107}$ V. Azzolini, ${ }^{107}$ A. Calamba, ${ }^{107}$ R. Carroll,,${ }^{107}$ T. Ferguson, ${ }^{107}$ Y. Iiyama, ${ }^{107}$ D. W. Jang, ${ }^{107}$ Y. F. Liu, ${ }^{107}$ M. Paulini,,${ }^{107}$ H. Vogel,,${ }^{107}$ I. Vorobiev,${ }^{107}$ J. P. Cumalat, ${ }^{108}$ B. R. Drell,,${ }^{108}$ 
C. J. Edelmaier, ${ }^{108}$ W. T. Ford ${ }^{108}$ A. Gaz,${ }^{108}$ B. Heyburn, ${ }^{108}$ E. Luiggi Lopez, ${ }^{108}$ J. G. Smith,${ }^{108}$ K. Stenson, ${ }^{108}$ K. A. Ulmer, ${ }^{108}$ S. R. Wagner, ${ }^{108}$ J. Alexander, ${ }^{109}$ A. Chatterjee, ${ }^{109}$ N. Eggert ${ }^{109}$ L. K. Gibbons, ${ }^{109}$ B. Heltsley, ${ }^{109}$ A. Khukhunaishvili, ${ }^{109}$ B. Kreis, ${ }^{109}$ N. Mirman, ${ }^{109}$ G. Nicolas Kaufman, ${ }^{109}$ J. R. Patterson, ${ }^{109}$ A. Ryd, ${ }^{109}$ E. Salvati, ${ }^{109}$ W. Sun, ${ }^{109}$ W. D. Teo, ${ }^{109}$ J. Thom, ${ }^{109}$ J. Thompson, ${ }^{109}$ J. Tucker,${ }^{109}$ J. Vaughan, ${ }^{109}$ Y. Weng, ${ }^{109}$ L. Winstrom, ${ }^{109}$ P. Wittich, ${ }^{109}$ D. Winn, ${ }^{10}$ S. Abdullin, ${ }^{111}$ M. Albrow, ${ }^{111}$ J. Anderson, ${ }^{111}$ L. A. T. Bauerdick, ${ }^{111}$

A. Beretvas, ${ }^{111}$ J. Berryhill, ${ }^{111}$ P. C. Bhat, ${ }^{111}$ I. Bloch, ${ }^{111}$ K. Burkett,${ }^{111}$ J. N. Butler, ${ }^{111}$ V. Chetluru, ${ }^{111}$ H. W. K. Cheung, ${ }^{111}$ F. Chlebana, ${ }^{111}$ V. D. Elvira, ${ }^{111}$ I. Fisk, ${ }^{111}$ J. Freeman, ${ }^{111}$ Y. Gao, ${ }^{111}$ D. Green, ${ }^{111}$ O. Gutsche,${ }^{111}$ J. Hanlon, ${ }^{111}$ R. M. Harris, ${ }^{111}$ J. Hirschauer, ${ }^{111}$ B. Hooberman, ${ }^{111}$ S. Jindariani, ${ }^{111}$ M. Johnson, ${ }^{111}$ U. Joshi, ${ }^{111}$ B. Kilminster, ${ }^{111}$ B. Klima, ${ }^{111}$ S. Kunori, ${ }^{111}$ S. Kwan, ${ }^{111}$ C. Leonidopoulos,${ }^{111}$ J. Linacre, ${ }^{111}$ D. Lincoln,,${ }^{111}$ R. Lipton, ${ }^{111}$ J. Lykken, ${ }^{111}$ K. Maeshima, ${ }^{111}$ J. M. Marraffino, ${ }^{111}$ S. Maruyama, ${ }^{111}$ D. Mason, ${ }^{111}$ P. McBride,${ }^{111}$

K. Mishra, ${ }^{111}$ S. Mrenna, ${ }^{111}$ Y. Musienko, ${ }^{11, \text { aaa }}$ C. Newman-Holmes, ${ }^{111}$ V. O’Dell, ${ }^{111}$ O. Prokofyev, ${ }^{111}$

E. Sexton-Kennedy, ${ }^{111}$ S. Sharma,${ }^{111}$ W. J. Spalding,${ }^{111}$ L. Spiegel, ${ }^{111}$ P. Tan,${ }^{111}$ L. Taylor,${ }^{111}$ S. Tkaczyk,${ }^{111}$ N. V. Tran, ${ }^{111}$ L. Uplegger, ${ }^{111}$ E. W. Vaandering, ${ }^{111}$ R. Vidal, ${ }^{111}$ J. Whitmore,,${ }^{111}$ W. Wu, ${ }^{111}$ F. Yang, ${ }^{111}$ F. Yumiceva, ${ }^{111}$ J. C. Yun, ${ }^{111}$ D. Acosta, ${ }^{112}$ P. Avery, ${ }^{112}$ D. Bourilkov, ${ }^{112}$ M. Chen, ${ }^{112}$ T. Cheng, ${ }^{112}$ S. Das, ${ }^{112}$ M. De Gruttola, ${ }^{112}$ G. P. Di Giovanni, ${ }^{12}$ D. Dobur, ${ }^{112}$ A. Drozdetskiy, ${ }^{112}$ R. D. Field, ${ }^{112}$ M. Fisher, ${ }^{12}$ Y. Fu, ${ }^{112}$

I. K. Furic, ${ }^{112}$ J. Gartner, ${ }^{12}$ J. Hugon, ${ }^{12}$ B. Kim, ${ }^{112}$ J. Konigsberg, ${ }^{112}$ A. Korytov, ${ }^{112}$ A. Kropivnitskaya, ${ }^{112}$ T. Kypreos, ${ }^{112}$ J. F. Low, ${ }^{112}$ K. Matchev, ${ }^{112}$ P. Milenovic, ${ }^{112, b b b}$ G. Mitselmakher, ${ }^{112}$ L. Muniz, ${ }^{112}$ R. Remington, ${ }^{112}$ A. Rinkevicius, ${ }^{112}$ P. Sellers, ${ }^{112}$ N. Skhirtladze, ${ }^{112}$ M. Snowball,${ }^{112}$ J. Yelton, ${ }^{112}$ M. Zakaria, ${ }^{112}$ V. Gaultney, ${ }^{113}$ S. Hewamanage, ${ }^{113}$ L. M. Lebolo, ${ }^{113}$ S. Linn, ${ }^{113}$ P. Markowitz,${ }^{113}$ G. Martinez,${ }^{113}$ J. L. Rodriguez, ${ }^{113}$ T. Adams,${ }^{114}$

A. Askew, ${ }^{114}$ J. Bochenek, ${ }^{114}$ J. Chen, ${ }^{114}$ B. Diamond, ${ }^{114}$ S. V. Gleyzer, ${ }^{114}$ J. Haas, ${ }^{114}$ S. Hagopian, ${ }^{114}$ V. Hagopian, ${ }^{114}$ M. Jenkins, ${ }^{114}$ K. F. Johnson, ${ }^{114}$ H. Prosper, ${ }^{114}$ V. Veeraraghavan, ${ }^{114}$ M. Weinberg, ${ }^{114}$ M. M. Baarmand, ${ }^{115}$ B. Dorney, ${ }^{115}$ M. Hohlmann, ${ }^{115}$ H. Kalakhety, ${ }^{115}$ I. Vodopiyanov, ${ }^{115}$ M. R. Adams, ${ }^{116}$ I. M. Anghel, ${ }^{116}$ L. Apanasevich, ${ }^{116}$ Y. Bai, ${ }^{116}$ V. E. Bazterra, ${ }^{116}$ R. R. Betts, ${ }^{116}$ I. Bucinskaite, ${ }^{116}$ J. Callner, ${ }^{116}$ R. Cavanaugh, ${ }^{116}$ O. Evdokimov, ${ }^{116}$ L. Gauthier, ${ }^{116}$ C. E. Gerber, ${ }^{116}$ D. J. Hofman, ${ }^{116}$ S. Khalatyan, ${ }^{116}$ F. Lacroix, ${ }^{116}$

M. Malek, ${ }^{116}$ C. O'Brien, ${ }^{116}$ C. Silkworth, ${ }^{116}$ D. Strom, ${ }^{116}$ N. Varelas, ${ }^{116}$ U. Akgun, ${ }^{117}$ E. A. Albayrak, ${ }^{117}$ B. Bilki, ${ }^{117, \text { ccc }}$ W. Clarida, ${ }^{117}$ F. Duru, ${ }^{117}$ S. Griffiths, ${ }^{117}$ J.-P. Merlo, ${ }^{117}$ H. Mermerkaya, ${ }^{117, \text { ddd }}$ A. Mestvirishvili, ${ }^{117}$ A. Moeller ${ }^{117}$ J. Nachtman, ${ }^{117}$ C. R. Newsom, ${ }^{117}$ E. Norbeck, ${ }^{117}$ Y. Onel, ${ }^{117}$ F. Ozok, ${ }^{117}$ S. Sen, ${ }^{117}$ E. Tiras, ${ }^{117}$ J. Wetzel, ${ }^{117}$ T. Yetkin, ${ }^{117}$ K. Yi, ${ }^{117}$ B. A. Barnett, ${ }^{118}$ B. Blumenfeld, ${ }^{118}$ S. Bolognesi, ${ }^{118}$ D. Fehling, ${ }^{118}$ G. Giurgiu, ${ }^{118}$

A. V. Gritsan, ${ }^{118}$ Z. J. Guo, ${ }^{118}$ G. Hu, ${ }^{118}$ P. Maksimovic, ${ }^{118}$ S. Rappoccio, ${ }^{118}$ M. Swartz, ${ }^{118}$ A. Whitbeck, ${ }^{118}$ P. Baringer, ${ }^{119}$ A. Bean, ${ }^{119}$ G. Benelli, ${ }^{119}$ O. Grachov, ${ }^{119}$ R. P. Kenny Iii, ${ }^{119}$ M. Murray, ${ }^{119}$ D. Noonan,${ }^{119}$ S. Sanders, ${ }^{119}$ R. Stringer, ${ }^{119}$ G. Tinti, ${ }^{119}$ J. S. Wood, ${ }^{119}$ V. Zhukova, ${ }^{119}$ A. F. Barfuss, ${ }^{120}$ T. Bolton, ${ }^{120}$ I. Chakaberia, ${ }^{120}$ A. Ivanov, ${ }^{120}$ S. Khalil, ${ }^{120}$ M. Makouski ${ }^{120}$ Y. Maravin, ${ }^{120}$ S. Shrestha, ${ }^{120}$ I. Svintradze, ${ }^{120}$ J. Gronberg, ${ }^{121}$ D. Lange, ${ }^{121}$ D. Wright, ${ }^{121}$ A. Baden, ${ }^{122}$ M. Boutemeur, ${ }^{122}$ B. Calvert, ${ }^{122}$ S. C. Eno, ${ }^{122}$ J. A. Gomez, ${ }^{122}$ N. J. Hadley, ${ }^{122}$ R. G. Kellogg, ${ }^{122}$ M. Kirn, ${ }^{122}$ T. Kolberg, ${ }^{122}$ Y. Lu, ${ }^{122}$ M. Marionneau, ${ }^{122}$ A. C. Mignerey, ${ }^{122}$ K. Pedro, ${ }^{122}$ A. Peterman, ${ }^{122}$ A. Skuja, ${ }^{122}$ J. Temple, ${ }^{122}$ M. B. Tonjes, ${ }^{122}$ S. C. Tonwar, ${ }^{122}$ E. Twedt ${ }^{122}$ A. Apyan, ${ }^{123}$ G. Bauer, ${ }^{123}$ J. Bendavid, ${ }^{123}$ W. Busza, ${ }^{123}$ E. Butz, ${ }^{123}$ I. A. Cali, ${ }^{123}$ M. Chan, ${ }^{123}$ V. Dutta, ${ }^{123}$ G. Gomez Ceballos, ${ }^{123}$ M. Goncharov, ${ }^{123}$ K. A. Hahn, ${ }^{123}$ Y. Kim, ${ }^{123}$ M. Klute, ${ }^{123}$ K. Krajczar, ${ }^{123, \text { eee }}$ W. Li,${ }^{123}$ P. D. Luckey, ${ }^{123}$ T. Ma, ${ }^{123}$ S. Nahn, ${ }^{123}$ C. Paus, ${ }^{123}$ D. Ralph, ${ }^{123}$ C. Roland, ${ }^{123}$ G. Roland, ${ }^{123}$ M. Rudolph,${ }^{123}$ G. S. F. Stephans, ${ }^{123}$ F. Stöckli, ${ }^{123}$ K. Sumorok, ${ }^{123}$ K. Sung, ${ }^{123}$ D. Velicanu, ${ }^{123}$ E. A. Wenger, ${ }^{123}$ R. Wolf,${ }^{123}$

B. Wyslouch, ${ }^{123}$ M. Yang, ${ }^{123}$ Y. Yilmaz, ${ }^{123}$ A. S. Yoon, ${ }^{123}$ M. Zanetti, ${ }^{123}$ S. I. Cooper, ${ }^{124}$ B. Dahmes, ${ }^{124}$

A. De Benedetti, ${ }^{124}$ G. Franzoni, ${ }^{124}$ A. Gude ${ }^{124}$ S. C. Kao, ${ }^{124}$ K. Klapoetke, ${ }^{124}$ Y. Kubota, ${ }^{124}$ J. Mans, ${ }^{124}$ N. Pastika, ${ }^{124}$ R. Rusack, ${ }^{124}$ M. Sasseville, ${ }^{124}$ A. Singovsky, ${ }^{124}$ N. Tambe, ${ }^{124}$ J. Turkewitz, ${ }^{124}$ L. M. Cremaldi, ${ }^{125}$ R. Kroeger, ${ }^{125}$ L. Perera, ${ }^{125}$ R. Rahmat, ${ }^{125}$ D. A. Sanders, ${ }^{125}$ E. Avdeeva, ${ }^{126}$ K. Bloom, ${ }^{126}$ S. Bose, ${ }^{126}$ J. Butt, ${ }^{126}$ D. R. Claes, ${ }^{126}$ A. Dominguez, ${ }^{126}$ M. Eads, ${ }^{126}$ J. Keller, ${ }^{126}$ I. Kravchenko, ${ }^{126}$ J. Lazo-Flores, ${ }^{126}$ H. Malbouisson, ${ }^{126}$ S. Malik, ${ }^{126}$ G. R. Snow, ${ }^{126}$ U. Baur, ${ }^{127}$ A. Godshalk, ${ }^{127}$ I. Iashvili, ${ }^{127}$ S. Jain, ${ }^{127}$ A. Kharchilava, ${ }^{127}$ A. Kumar, ${ }^{127}$ S. P. Shipkowski, ${ }^{127}$ K. Smith, ${ }^{127}$ G. Alverson, ${ }^{128}$ E. Barberis, ${ }^{128}$ D. Baumgartel, ${ }^{128}$ M. Chasco, ${ }^{128}$ J. Haley, ${ }^{128}$ D. Nash, ${ }^{128}$ D. Trocino, ${ }^{128}$ D. Wood, ${ }^{128}$ J. Zhang, ${ }^{128}$ A. Anastassov, ${ }^{129}$ A. Kubik, ${ }^{129}$ N. Mucia, ${ }^{129}$ N. Odell, ${ }^{129}$ R. A. Ofierzynski, ${ }^{129}$ B. Pollack, ${ }^{129}$ A. Pozdnyakov, ${ }^{129}$ M. Schmitt, ${ }^{129}$ S. Stoynev, ${ }^{129}$ M. Velasco, ${ }^{129}$ S. Won ${ }^{129}$ L. Antonelli, ${ }^{130}$ D. Berry, ${ }^{130}$ A. Brinkerhoff, ${ }^{130}$ M. Hildreth, ${ }^{130}$ C. Jessop, ${ }^{130}$ D. J. Karmgard, ${ }^{130}$ J. Kolb, ${ }^{130}$ K. Lannon, ${ }^{130}$ W. Luo, ${ }^{130}$ S. Lynch, ${ }^{130}$ N. Marinelli, ${ }^{130}$ D. M. Morse, ${ }^{130}$ T. Pearson, ${ }^{130}$ M. Planer, ${ }^{130}$ R. Ruchti, ${ }^{130}$ J. Slaunwhite, ${ }^{130}$ N. Valls, ${ }^{130}$ M. Wayne, ${ }^{130}$ M. Wolf,${ }^{130}$ B. Bylsma, ${ }^{131}$ L. S. Durkin, ${ }^{131}$ C. Hill,${ }^{131}$ R. Hughes, ${ }^{131}$ 
R. Hughes, ${ }^{131}$ K. Kotov, ${ }^{131}$ T. Y. Ling, ${ }^{131}$ D. Puigh,,${ }^{131}$ M. Rodenburg,,${ }^{131}$ C. Vuosalo, ${ }^{131}$ G. Williams, ${ }^{131}$ B. L. Winer, ${ }^{131}$ N. Adam, ${ }^{132}$ E. Berry ${ }^{132}$ P. Elmer, ${ }^{132}$ D. Gerbaudo, ${ }^{132}$ V. Halyo, ${ }^{132}$ P. Hebda, ${ }^{132}$ J. Hegeman, ${ }^{132}$ A. Hunt, ${ }^{132}$ P. Jindal, ${ }^{132}$ D. Lopes Pegna, ${ }^{132}$ P. Lujan, ${ }^{132}$ D. Marlow, ${ }^{132}$ T. Medvedeva, ${ }^{132}$ M. Mooney,,${ }^{132}$ J. Olsen, ${ }^{132}$ P. Piroué, ${ }^{132}$ X. Quan, ${ }^{132}$ A. Raval, ${ }^{132}$ B. Safdi, ${ }^{132}$ H. Saka, ${ }^{132}$ D. Stickland, ${ }^{132}$ C. Tully, ${ }^{132}$ J. S. Werner, ${ }^{132}$ A. Zuranski, ${ }^{132}$ J. G. Acosta, ${ }^{133}$ E. Brownson, ${ }^{133}$ X. T. Huang, ${ }^{133}$ A. Lopez ${ }^{133}$ H. Mendez, ${ }^{133}$ S. Oliveros,${ }^{133}$

J.E. Ramirez Vargas, ${ }^{133}$ A. Zatserklyaniy, ${ }^{133}$ E. Alagoz, ${ }^{134}$ V. E. Barnes,${ }^{134}$ D. Benedetti, ${ }^{134}$ G. Bolla, ${ }^{134}$ D. Bortoletto, ${ }^{134}$ M. De Mattia, ${ }^{134}$ A. Everett, ${ }^{134}$ Z. Hu, ${ }^{134}$ M. Jones,,${ }^{134}$ O. Koybasi, ${ }^{134}$ M. Kress, ${ }^{134}$ A. T. Laasanen, ${ }^{134}$ N. Leonardo, ${ }^{134}$ V. Maroussov, ${ }^{134}$ P. Merkel, ${ }^{134}$ D. H. Miller, ${ }^{134}$ N. Neumeister, ${ }^{134}$ I. Shipsey, ${ }^{134}$ D. Silvers, ${ }^{134}$ A. Svyatkovskiy, ${ }^{134}$ M. Vidal Marono, ${ }^{134}$ H. D. Yoo, ${ }^{134}$ J. Zablocki, ${ }^{134}$ Y. Zheng, ${ }^{134}$ S. Guragain, ${ }^{135}$ N. Parashar, ${ }^{135}$ A. Adair, ${ }^{136}$ C. Boulahouache, ${ }^{136}$ K. M. Ecklund, ${ }^{136}$ F. J. M. Geurts, ${ }^{136}$ B. P. Padley, ${ }^{136}$ R. Redjimi, ${ }^{136}$ J. Roberts, ${ }^{136}$ J. Zabel, ${ }^{136}$ B. Betchart, ${ }^{137}$ A. Bodek,,${ }^{137}$ Y. S. Chung, ${ }^{137}$ R. Covarelli, ${ }^{137}$ P. de Barbaro, ${ }^{137}$

R. Demina, ${ }^{137}$ Y. Eshaq, ${ }^{137}$ A. Garcia-Bellido, ${ }^{137}$ P. Goldenzweig, ${ }^{137}$ J. Han ${ }^{137}$ A. Harel, ${ }^{137}$ D. C. Miner, ${ }^{137}$ D. Vishnevskiy, ${ }^{137}$ M. Zielinski, ${ }^{137}$ A. Bhatti, ${ }^{138}$ R. Ciesielski, ${ }^{138}$ L. Demortier, ${ }^{138}$ K. Goulianos, ${ }^{138}$ G. Lungu, ${ }^{138}$ S. Malik, ${ }^{138}$ C. Mesropian, ${ }^{138}$ S. Arora, ${ }^{139}$ A. Barker, ${ }^{139}$ J. P. Chou,${ }^{139}$ C. Contreras-Campana, ${ }^{139}$

E. Contreras-Campana, ${ }^{139}$ D. Duggan, ${ }^{139}$ D. Ferencek, ${ }^{139}$ Y. Gershtein, ${ }^{139}$ R. Gray, ${ }^{139}$ E. Halkiadakis, ${ }^{139}$ D. Hidas, ${ }^{139}$ A. Lath, ${ }^{139}$ S. Panwalkar, ${ }^{139}$ M. Park, ${ }^{139}$ R. Patel, ${ }^{139}$ V. Rekovic,,${ }^{139}$ J. Robles, ${ }^{139}$ K. Rose, ${ }^{139}$ S. Salur, ${ }^{139}$ S. Schnetzer, ${ }^{139}$ C. Seitz, ${ }^{139}$ S. Somalwar, ${ }^{139}$ R. Stone, ${ }^{139}$ S. Thomas, ${ }^{139}$ G. Cerizza, ${ }^{140}$ M. Hollingsworth, ${ }^{140}$ S. Spanier, ${ }^{140}$ Z. C. Yang, ${ }^{140}$ A. York, ${ }^{140}$ R. Eusebi,${ }^{141}$ W. Flanagan, ${ }^{141}$ J. Gilmore, ${ }^{141}$ T. Kamon, ${ }^{141, f f f}$ V. Khotilovich, ${ }^{141}$ R. Montalvo, ${ }^{141}$ I. Osipenkov, ${ }^{141}$ Y. Pakhotin, ${ }^{141}$ A. Perloff, ${ }^{141}$ J. Roe,${ }^{141}$ A. Safonov, ${ }^{141}$ T. Sakuma, ${ }^{141}$ S. Sengupta, ${ }^{141}$ I. Suarez, ${ }^{141}$ A. Tatarinov, ${ }^{141}$ D. Toback, ${ }^{141}$ N. Akchurin, ${ }^{142}$ J. Damgov, ${ }^{142}$ C. Dragoiu, ${ }^{142}$ P. R. Dudero, ${ }^{142}$ C. Jeong, ${ }^{142}$ K. Kovitanggoon, ${ }^{142}$ S. W. Lee, ${ }^{142}$ T. Libeiro, ${ }^{142}$ Y. Roh, ${ }^{142}$ I. Volobouev, ${ }^{142}$ E. Appelt, ${ }^{143}$ A. G. Delannoy, ${ }^{143}$ C. Florez, ${ }^{143}$ S. Greene, ${ }^{143}$ A. Gurrola, ${ }^{143}$ W. Johns, ${ }^{143}$ C. Johnston, ${ }^{143}$ P. Kurt, ${ }^{143}$ C. Maguire, ${ }^{143}$ A. Melo, ${ }^{143}$ M. Sharma,${ }^{143}$ P. Sheldon, ${ }^{143}$ B. Snook, ${ }^{143}$ S. Tuo, ${ }^{143}$ J. Velkovska, ${ }^{143}$ M. W. Arenton, ${ }^{144}$ M. Balazs, ${ }^{144}$ S. Boutle, ${ }^{144}$ B. Cox ${ }^{144}$ B. Francis,${ }^{144}$ J. Goodell, ${ }^{144}$ R. Hirosky, ${ }^{144}$ A. Ledovskoy, ${ }^{144}$ C. Lin, ${ }^{144}$ C. Neu ${ }^{144}$ J. Wood,${ }^{144}$ R. Yohay, ${ }^{144}$ S. Gollapinni, ${ }^{145}$ R. Harr, ${ }^{145}$ P. E. Karchin, ${ }^{145}$

C. Kottachchi Kankanamge Don, ${ }^{145}$ P. Lamichhane, ${ }^{145}$ A. Sakharov,${ }^{145}$ M. Anderson, ${ }^{146}$ D. Belknap ${ }^{146}$ L. Borrello, ${ }^{146}$ D. Carlsmith, ${ }^{146}$ M. Cepeda, ${ }^{146}$ S. Dasu, ${ }^{146}$ E. Friis, ${ }^{146}$ L. Gray, ${ }^{146}$ K. S. Grogg, ${ }^{146}$ M. Grothe, ${ }^{146}$ R. Hall-Wilton, ${ }^{146}$ M. Herndon, ${ }^{146}$ A. Hervé, ${ }^{146}$ P. Klabbers, ${ }^{146}$ J. Klukas, ${ }^{146}$ A. Lanaro, ${ }^{146}$ C. Lazaridis, ${ }^{146}$ J. Leonard, ${ }^{146}$ R. Loveless, ${ }^{146}$ A. Mohapatra, ${ }^{146}$ I. Ojalvo, ${ }^{146}$ F. Palmonari, ${ }^{146}$ G. A. Pierro, ${ }^{146}$ I. Ross, ${ }^{146}$ A. Savin, ${ }^{146}$ W. H. Smith, ${ }^{146}$ and J. Swanson ${ }^{146}$

\title{
(CMS Collaboration)
}

\author{
${ }^{1}$ Yerevan Physics Institute, Yerevan, Armenia \\ ${ }^{2}$ Institut für Hochenergiephysik der OeAW, Wien, Austria \\ ${ }^{3}$ National Centre for Particle and High Energy Physics, Minsk, Belarus \\ ${ }^{4}$ Universiteit Antwerpen, Antwerpen, Belgium \\ ${ }^{5}$ Vrije Universiteit Brussel, Brussel, Belgium \\ ${ }^{6}$ Université Libre de Bruxelles, Bruxelles, Belgium \\ ${ }^{7}$ Ghent University, Ghent, Belgium \\ ${ }^{8}$ Université Catholique de Louvain, Louvain-la-Neuve, Belgium \\ ${ }^{9}$ Université de Mons, Mons, Belgium \\ ${ }^{10}$ Centro Brasileiro de Pesquisas Fisicas, Rio de Janeiro, Brazil \\ ${ }^{11}$ Universidade do Estado do Rio de Janeiro, Rio de Janeiro, Brazil \\ ${ }^{12}$ Instituto de Fisica Teorica, Universidade Estadual Paulista, Sao Paulo, Brazil \\ ${ }^{13}$ Institute for Nuclear Research and Nuclear Energy, Sofia, Bulgaria \\ ${ }^{14}$ University of Sofia, Sofia, Bulgaria \\ ${ }^{15}$ Institute of High Energy Physics, Beijing, China \\ ${ }^{16}$ State Key Laboratory of Nuclear Physics and Technology, Peking University, Beijing, China \\ ${ }^{17}$ Universidad de Los Andes, Bogota, Colombia \\ ${ }^{18}$ Technical University of Split, Split, Croatia \\ ${ }^{19}$ University of Split, Split, Croatia \\ ${ }^{20}$ Institute Rudjer Boskovic, Zagreb, Croatia \\ ${ }^{21}$ University of Cyprus, Nicosia, Cyprus \\ ${ }^{22}$ Charles University, Prague, Czech Republic
}


${ }^{23}$ Academy of Scientific Research and Technology of the Arab Republic of Egypt,

Egyptian Network of High Energy Physics, Cairo, Egypt

${ }^{24}$ National Institute of Chemical Physics and Biophysics, Tallinn, Estonia

${ }^{25}$ Department of Physics, University of Helsinki, Helsinki, Finland

${ }^{26}$ Helsinki Institute of Physics, Helsinki, Finland

${ }^{27}$ Lappeenranta University of Technology, Lappeenranta, Finland

${ }^{28}$ DSM/IRFU, CEA/Saclay, Gif-sur-Yvette, France

${ }^{29}$ Laboratoire Leprince-Ringuet, Ecole Polytechnique, IN2P3-CNRS, Palaiseau, France

${ }^{30}$ Institut Pluridisciplinaire Hubert Curien, Université de Strasbourg, Université de Haute Alsace Mulhouse, CNRS/IN2P3, Strasbourg, France

${ }^{31}$ Centre de Calcul de l'Institut National de Physique Nucleaire et de Physique des Particules,

CNRS/IN2P3, Villeurbanne, France, Villeurbanne, France

${ }^{32}$ Université de Lyon, Université Claude Bernard Lyon 1, CNRS-IN2P3, Institut de Physique Nucléaire de Lyon, Villeurbanne, France

${ }^{33}$ Institute of High Energy Physics and Informatization, Tbilisi State University, Tbilisi, Georgia

${ }^{34}$ RWTH Aachen University, I. Physikalisches Institut, Aachen, Germany

${ }^{35}$ RWTH Aachen University, III. Physikalisches Institut A, Aachen, Germany

${ }^{36}$ RWTH Aachen University, III. Physikalisches Institut B, Aachen, Germany

${ }^{37}$ Deutsches Elektronen-Synchrotron, Hamburg, Germany

${ }^{38}$ University of Hamburg, Hamburg, Germany

${ }^{39}$ Institut für Experimentelle Kernphysik, Karlsruhe, Germany

${ }^{40}$ Institute of Nuclear Physics “Demokritos”, Aghia Paraskevi, Greece

${ }^{41}$ University of Athens, Athens, Greece

${ }^{42}$ University of Ioánnina, Ioánnina, Greece

${ }^{43}$ KFKI Research Institute for Particle and Nuclear Physics, Budapest, Hungary

${ }^{44}$ Institute of Nuclear Research ATOMKI, Debrecen, Hungary

${ }^{45}$ University of Debrecen, Debrecen, Hungary

${ }^{46}$ Panjab University, Chandigarh, India

${ }^{47}$ University of Delhi, Delhi, India

${ }^{48}$ Saha Institute of Nuclear Physics, Kolkata, India

${ }^{49}$ Bhabha Atomic Research Centre, Mumbai, India

${ }^{50}$ Tata Institute of Fundamental Research - EHEP, Mumbai, India

${ }^{51}$ Tata Institute of Fundamental Research - HECR, Mumbai, India

${ }^{52}$ Institute for Research in Fundamental Sciences (IPM), Tehran, Iran

${ }^{53}$ INFN Sezione di Bari, Bari, Italy

${ }^{53 \mathrm{~b}}$ Università di Bari, Bari, Italy

${ }^{53 \mathrm{c}}$ Politecnico di Bari, Bari, Italy

${ }^{54 a}$ INFN Sezione di Bologna, Bologna, Italy

${ }^{54 \mathrm{~b}}$ Università di Bologna, Bologna, Italy

${ }^{55 a}$ INFN Sezione di Catania, Catania, Italy

${ }^{55 \mathrm{~b}}$ Università di Catania, Catania, Italy

${ }^{56 a}$ INFN Sezione di Firenze, Firenze, Italy

${ }^{56 \mathrm{~b}}$ Università di Firenze, Firenze, Italy

${ }^{57}$ INFN Laboratori Nazionali di Frascati, Frascati, Italy

${ }^{58 a}$ INFN Sezione di Genova, Genova, Italy

${ }^{58 \mathrm{~b}}$ Università di Genova, Genova, Italy

${ }^{59 a}$ INFN Sezione di Milano-Bicocca, Milano, Italy

${ }^{59 \mathrm{~b}}$ Università di Milano-Bicocca, Milano, Italy

${ }^{60 a}$ INFN Sezione di Napoli, Napoli, Italy

${ }^{60 \mathrm{~b}}$ Università di Napoli "Federico II," Napoli, Italy

61a INFN Sezione di Padova, Padova, Italy

${ }^{61 \mathrm{~b}}$ Università di Padova, Padova, Italy

${ }^{61 \mathrm{c}}$ Università di Trento (Trento), Padova, Italy

${ }^{62 \mathrm{a}}$ INFN Sezione di Pavia, Pavia, Italy

${ }^{62 \mathrm{~b}}$ Università di Pavia, Pavia, Italy

${ }^{63 \mathrm{a}}$ INFN Sezione di Perugia, Perugia, Italy

${ }^{63 \mathrm{~b}}$ Università di Perugia, Perugia, Italy

${ }^{64 a}$ INFN Sezione di Pisa, Pisa, Italy

${ }^{64 \mathrm{~b}}$ Università di Pisa, Pisa, Italy

${ }^{64 \mathrm{c}}$ Scuola Normale Superiore di Pisa, Pisa, Italy

${ }^{65}$ INFN Sezione di Roma, Roma, Italy

${ }^{65 b}$ Università di Roma “La Sapienza," Roma, Italy 
${ }^{66 a}$ INFN Sezione di Torino, Torino, Italy

${ }^{66 \mathrm{~b}}$ Università di Torino, Torino, Italy

${ }^{66 \mathrm{c}}$ Università del Piemonte Orientale (Novara), Torino, Italy

${ }^{67 a}$ INFN Sezione di Trieste, Trieste, Italy

${ }^{67 \mathrm{~b}}$ Università di Trieste, Trieste, Italy

${ }^{53}$ Kangwon National University, Chunchon, Korea

${ }^{54}$ Kyungpook National University, Daegu, Korea

${ }^{55}$ Chonnam National University, Institute for Universe and Elementary Particles, Kwangju, Korea

${ }^{56}$ Korea University, Seoul, Korea

${ }^{57}$ University of Seoul, Seoul, Korea

${ }^{58}$ Sungkyunkwan University, Suwon, Korea

${ }^{59}$ Vilnius University, Vilnius, Lithuania

${ }^{60}$ Centro de Investigacion y de Estudios Avanzados del IPN, Mexico City, Mexico

${ }^{61}$ Universidad Iberoamericana, Mexico City, Mexico

${ }^{62}$ Benemerita Universidad Autonoma de Puebla, Puebla, Mexico

${ }^{63}$ Universidad Autónoma de San Luis Potosí, San Luis Potosí, Mexico

${ }^{64}$ University of Auckland, Auckland, New Zealand

${ }^{65}$ University of Canterbury, Christchurch, New Zealand

${ }^{66}$ National Centre for Physics, Quaid-I-Azam University, Islamabad, Pakistan

${ }^{67}$ National Centre for Nuclear Research, Swierk, Poland

${ }^{68}$ Institute of Experimental Physics, Faculty of Physics, University of Warsaw, Warsaw, Poland

${ }^{69}$ Laboratório de Instrumentação e Física Experimental de Partículas, Lisboa, Portugal

${ }^{70}$ Joint Institute for Nuclear Research, Dubna, Russia

${ }^{71}$ Petersburg Nuclear Physics Institute, Gatchina (St. Petersburg), Russia

${ }^{72}$ Institute for Nuclear Research, Moscow, Russia

${ }^{73}$ Institute for Theoretical and Experimental Physics, Moscow, Russia

${ }^{74}$ Moscow State University, Moscow, Russia

${ }^{75}$ P. N. Lebedev Physical Institute, Moscow, Russia

${ }^{76}$ State Research Center of Russian Federation, Institute for High Energy Physics, Protvino, Russia

${ }^{77}$ University of Belgrade, Faculty of Physics and Vinca Institute of Nuclear Sciences, Belgrade, Serbia

${ }^{78}$ Centro de Investigaciones Energéticas Medioambientales y Tecnológicas (CIEMAT), Madrid, Spain

${ }^{79}$ Universidad Autónoma de Madrid, Madrid, Spain

${ }^{80}$ Universidad de Oviedo, Oviedo, Spain

${ }^{81}$ Instituto de Física de Cantabria (IFCA), CSIC-Universidad de Cantabria, Santander, Spain

${ }^{82}$ CERN, European Organization for Nuclear Research, Geneva, Switzerland

${ }^{83}$ Paul Scherrer Institut, Villigen, Switzerland

${ }^{84}$ Institute for Particle Physics, ETH Zurich, Zurich, Switzerland

${ }^{85}$ Universität Zürich, Zurich, Switzerland

${ }^{86}$ National Central University, Chung-Li, Taiwan

${ }^{87}$ National Taiwan University (NTU), Taipei, Taiwan

${ }^{88}$ Cukurova University, Adana, Turkey

${ }^{89}$ Middle East Technical University, Physics Department, Ankara, Turkey

${ }^{90}$ Bogazici University, Istanbul, Turkey

${ }^{91}$ Istanbul Technical University, Istanbul, Turkey

${ }^{92}$ National Scientific Center, Kharkov Institute of Physics and Technology, Kharkov, Ukraine

${ }^{93}$ University of Bristol, Bristol, United Kingdom

${ }^{94}$ Rutherford Appleton Laboratory, Didcot, United Kingdom

${ }^{95}$ Imperial College, London, United Kingdom

${ }^{96}$ Brunel University, Uxbridge, United Kingdom

${ }^{97}$ Baylor University, Waco, Texas, USA

${ }^{98}$ The University of Alabama, Tuscaloosa, Alabama, USA

${ }^{99}$ Boston University, Boston, Massachusetts, USA

${ }^{100}$ Brown University, Providence, Rhode Island, USA

${ }^{101}$ University of California, Davis, Davis, California, USA

${ }^{102}$ University of California, Los Angeles, Los Angeles, USA

${ }^{103}$ University of California, Riverside, Riverside, California, USA

${ }^{104}$ University of California, San Diego, La Jolla, California, USA

${ }^{105}$ University of California, Santa Barbara, Santa Barbara, California, USA

${ }^{106}$ California Institute of Technology, Pasadena, California, USA

${ }^{107}$ Carnegie Mellon University, Pittsburgh, Pennsylvania, USA

${ }^{108}$ University of Colorado at Boulder, Boulder, Colorado, USA 
${ }^{109}$ Cornell University, Ithaca, New York, USA

${ }^{110}$ Fairfield University, Fairfield, Connecticut, USA

${ }^{111}$ Fermi National Accelerator Laboratory, Batavia, Illinois, USA

${ }^{112}$ University of Florida, Gainesville, Florida, USA

${ }^{113}$ Florida International University, Miami, Florida, USA

${ }^{114}$ Florida State University, Tallahassee, Florida, USA

${ }^{115}$ Florida Institute of Technology, Melbourne, Florida, USA

${ }^{116}$ University of Illinois at Chicago (UIC), Chicago, Illinois, USA

${ }^{117}$ The University of Iowa, Iowa City, Iowa, USA

${ }^{118}$ Johns Hopkins University, Baltimore, Maryland, USA

${ }^{119}$ The University of Kansas, Lawrence, Kansas, USA

${ }^{120}$ Kansas State University, Manhattan, Kansas, USA

${ }^{121}$ Lawrence Livermore National Laboratory, Livermore, California, USA

${ }^{122}$ University of Maryland, College Park, Maryland, USA

${ }^{123}$ Massachusetts Institute of Technology, Cambridge, Massachusetts, USA

${ }^{124}$ University of Minnesota, Minneapolis, Minnesota, USA

${ }^{125}$ University of Mississippi, Oxford, Mississippi, USA

${ }^{126}$ University of Nebraska-Lincoln, Lincoln, Nebraska, USA

${ }^{127}$ State University of New York at Buffalo, Buffalo, New York, USA

${ }^{128}$ Northeastern University, Boston, Massachusetts, USA

${ }^{129}$ Northwestern University, Evanston, Illinois, USA

${ }^{130}$ University of Notre Dame, Notre Dame, Indiana, USA

${ }^{131}$ The Ohio State University, Columbus, Ohio, USA

${ }^{132}$ Princeton University, Princeton, New Jersey, USA

${ }^{133}$ University of Puerto Rico, Mayaguez, Puerto Rico

${ }^{134}$ Purdue University, West Lafayette, Indiana, USA

${ }^{135}$ Purdue University Calumet, Hammond, Indiana, USA

${ }^{136}$ Rice University, Houston, Texas, USA

${ }^{137}$ University of Rochester, Rochester, New York, USA

${ }^{138}$ The Rockefeller University, New York, New York, USA

${ }^{139}$ Rutgers, The State University of New Jersey, Piscataway, New Jersey, USA

${ }^{140}$ University of Tennessee, Knoxville, Tennessee, USA

${ }^{141}$ Texas A\&M University, College Station, Texas, USA

${ }^{142}$ Texas Tech University, Lubbock, Texas, USA

${ }^{143}$ Vanderbilt University, Nashville, Tennessee, USA

${ }^{144}$ University of Virginia, Charlottesville, Virginia, USA

${ }^{145}$ Wayne State University, Detroit, Michigan, USA

${ }^{146}$ University of Wisconsin, Madison, Wisconsin, USA

${ }^{\mathrm{a}}$ Deceased.

${ }^{\mathrm{b}}$ Also at Vienna University of Technology, Vienna, Austria.

${ }^{\mathrm{c}}$ Also at National Institute of Chemical Physics and Biophysics, Tallinn, Estonia.

${ }^{\mathrm{d}}$ Also at Universidade Federal do ABC, Santo Andre, Brazil.

${ }^{\mathrm{e}}$ Also at California Institute of Technology, Pasadena, CA, USA.

${ }^{\mathrm{f}}$ Also at CERN, European Organization for Nuclear Research, Geneva, Switzerland.

${ }^{\mathrm{g}}$ Also at Laboratoire Leprince-Ringuet, Ecole Polytechnique, IN2P3-CNRS, Palaiseau, France.

${ }^{\mathrm{h}}$ Also at Suez Canal University, Suez, Egypt.

${ }^{\mathrm{i}}$ Also at Zewail City of Science and Technology, Zewail, Egypt.

${ }^{\mathrm{j}}$ Also at Cairo University, Cairo, Egypt.

${ }^{\mathrm{k}}$ Also at Fayoum University, El-Fayoum, Egypt.

${ }^{1}$ Also at British University, Cairo, Egypt.

${ }^{\mathrm{m}}$ Now at Ain Shams University, Cairo, Egypt.

${ }^{\mathrm{n}}$ Also at National Centre for Nuclear Research, Swierk, Poland.

${ }^{\circ}$ Also at Université de Haute-Alsace, Mulhouse, France.

${ }^{\mathrm{p}}$ Now at Joint Institute for Nuclear Research, Dubna, Russia.

${ }^{\mathrm{q}}$ Also at Moscow State University, Moscow, Russia.

${ }^{\mathrm{r}}$ Also at Brandenburg University of Technology, Cottbus, Germany.

${ }^{s}$ Also at Institute of Nuclear Research ATOMKI, Debrecen, Hungary.

${ }^{\mathrm{t}}$ Also at Eötvös Loránd University, Budapest, Hungary. 
${ }^{\mathrm{u}}$ Also at Tata Institute of Fundamental Research - HECR, Mumbai, India.

${ }^{\mathrm{v}}$ Also at University of Visva-Bharati, Santiniketan, India.

${ }^{\mathrm{w}}$ Also at Sharif University of Technology, Tehran, Iran.

${ }^{\mathrm{x}}$ Also at Isfahan University of Technology, Isfahan, Iran.

${ }^{\mathrm{y}}$ Also at Plasma Physics Research Center, Science and Research Branch, Islamic Azad University, Teheran, Iran.

${ }^{z}$ Also at Facoltà Ingegneria Università di Roma, Roma, Italy.

${ }^{\text {aa }}$ Also at Università della Basilicata, Potenza, Italy.

${ }^{\mathrm{bb}}$ Also at Università degli Studi Guglielmo Marconi, Roma, Italy.

${ }^{\mathrm{cc}}$ Also at Università degli Studi di Siena, Siena, Italy.

${ }^{\mathrm{dd}}$ Also at University of Bucharest, Faculty of Physics, Bucuresti-Magurele, Romania.

${ }^{e e}$ Also at Faculty of Physics of University of Belgrade, Belgrade, Serbia.

${ }^{\mathrm{ff}}$ Also at University of California, Los Angeles, Los Angeles, CA, USA.

${ }^{\mathrm{gg}}$ Also at Scuola Normale e Sezione dell' INFN, Pisa, Italy.

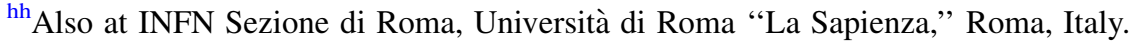

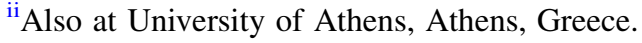

${ }^{\mathrm{jj}}$ Also at Rutherford Appleton Laboratory, Didcot, United Kingdom.

${ }^{\mathrm{kk}}$ Also at The University of Kansas, Lawrence, KS, USA.

${ }^{11}$ Also at Paul Scherrer Institut, Villigen, Switzerland.

${ }^{\mathrm{mm}}$ Also at Institute for Theoretical and Experimental Physics, Moscow, Russia.

${ }^{\mathrm{nn}}$ Also at Gaziosmanpasa University, Tokat, Turkey.

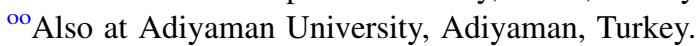

${ }^{\mathrm{pp}}$ Also at Izmir Institute of Technology, Izmir, Turkey.

${ }^{\mathrm{qq}} \mathrm{Also}$ at The University of Iowa, Iowa City, IA, USA.

${ }^{\mathrm{rr}}$ Also at Mersin University, Mersin, Turkey.

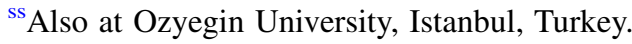

${ }^{t t}$ Also at Kafkas University, Kars, Turkey.

${ }^{\mathrm{uu}}$ Also at Suleyman Demirel University, Isparta, Turkey.

${ }^{\mathrm{vv}}$ Also at Ege University, Izmir, Turkey.

${ }^{w w}$ Also at School of Physics and Astronomy, University of Southampton, Southampton, United Kingdom.

${ }^{\mathrm{xx}}$ Also at INFN Sezione di Perugia, Università di Perugia, Perugia, Italy.

${ }^{\text {yy }}$ Also at University of Sydney, Sydney, Australia.

${ }^{\mathrm{zz}}$ Also at Utah Valley University, Orem, UT, USA.

${ }^{\text {aaa }}$ Also at Institute for Nuclear Research, Moscow, Russia.

${ }^{\mathrm{bbb}}$ Also at University of Belgrade, Faculty of Physics and Vinca Institute of Nuclear Sciences, Belgrade, Serbia.

${ }^{\mathrm{ccc}}$ Also at Argonne National Laboratory, Argonne, IL, USA.

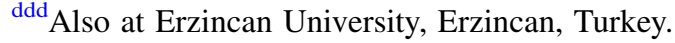

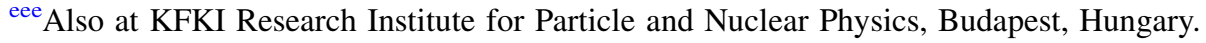

${ }^{\mathrm{fff}}$ Also at Kyungpook National University, Daegu, Korea. 